\title{
Completeness Rules for Spin Observables in Pseudoscalar Meson Photoproduction
}

\author{
Wen-Tai Chiang and Frank Tabakint \\ Department of Physics and Astronomy, University of Pittsburgh, Pittsburgh, PA 15260
}

(September 29, 2018)

\begin{abstract}
The number and type of measurements needed to ascertain the amplitudes for pseudoscalar meson photoproduction are analyzed in this paper. It is found that 8 carefully selected measurements can determine the four transversity amplitudes without discrete ambiguities. That number of measurements is one less than previously believed. We approach this problem in two distinct ways: (1) solving for the amplitude magnitudes and phases directly; and (2) using a bilinear helicity product formulation to map an algebra of measurements over to the well-known algebra of the $4 \times 4$ Gamma matrices. It is shown that the latter method leads to an alternate proof that 8 carefully chosen experiments suffice for determining the transversity amplitudes completely. In addition, Fierz transformations of the Gamma matrices are used to develop useful linear and nonlinear relationships between the spin observables. These relationships not only help in finding complete sets of experiments, but also yield important constraints between the 16 observables for this reaction.
\end{abstract}

PACS numbers: 11.80.Cr, 13.60.Le, 13.88.+e, 24.70.+s, 25.20.Lj 


\section{INTRODUCTION}

Interest in the photoproduction of pseudoscalar mesons has been revived now that experiments of unprecedented precision are imminent. With the development of new electron accelerator facilities (such as TJNAF) along with both polarized beams and targets and with the CLAS detector, it will soon be possible to measure various spin observables with precision. These observables include the differential cross section, $\sigma(\theta)$, plus three single spin observables $(\Sigma, T$ and $P$ ), which we denote as Type $\mathcal{S}$ measurements. In addition, there are twelve double spin observables which can be classified into three types: beam-target $(\mathcal{B T})$, beam-recoil $(\mathcal{B R})$ and target-recoil $(\mathcal{T} \mathcal{R})$ spin observables. The classic Barker, Donnachie and Storrow (BDS) [1] paper is one of the standard references on how to select measurements to fully determine the four (complex) total pseudoscalar meson photoproduction amplitudes. In this paper, we also address that question.

It is well-known that, without considering discrete ambiguities, seven measurement are needed to determine the four helicity amplitudes (four magnitudes plus three phases) up to an arbitrary overall phase. However, it is necessary to resolve all discrete ambiguities to extract complete information from experiments. In BDS, the following rule [1] (herein called the BDS rule) was promulgated:

In order to determine all amplitudes without discrete ambiguities, one has to measure five double spin observables along with the four type $\mathcal{S}$ measurements, provided no four double spin observables are selected from the same set of $\mathcal{B} \mathcal{T}$, $\mathcal{B R}$ and $\mathcal{T} \mathcal{R}$.

Thus, they say nine experiments are required.

Recently, Keaton and Workman (KW) [2] argued that selecting a complete set of observables is more complicated than the above BDS rule. However, KW were not able to provide sufficient conditions for resolving all ambiguities. Their work inspired us to investigate the problem of determining which experiments can provide a "complete set," e.g. those 
experiments which suffice to determine the basic amplitudes free of discrete or continuous ambiguities. Here we confirm the KW result [2] that there are cases obeying the BDS rule that still leave unresolved ambiguities. To our surprise, we also find that four appropriately chosen double spin observables, along with the four Type $\mathcal{S}$ measurements suffice to resolve all ambiguities. This is our major result. It is illustrated first by using the explicit approach used in BDS; namely, by solving for the magnitudes and phases of transversity helicity amplitudes. Transversity amplitudes provide the advantage of having all Type $\mathcal{S}$ (single spin) observables expressed in terms of the amplitude magnitudes only. The double spin observables are then needed to determine the phases of the transversity amplitudes.

Another approach is also provided in this paper. In this alternate approach, Hermitian versions of the usual $4 \times 4$ Gamma matrices are used to express all observables as bilinear products of helicity amplitudes. In that way, algebraic relations between observables (an algebra of measurements) are mapped into the well-known algebra of the $4 \times 4$ Gamma matrices. For example, important relationships between spin observables are derived here by applying the Fierz identities to products of Gamma matrices. This procedure, as explained later, yields useful relationships between observables which serve to select complete sets of observables. One benefit of this bilinear helicity product (BHP) approach is that it can be generalized to other reactions [3].

In Section II, we present the bilinear helicity product analysis of spin observables. In Section III, we give a general discussion of the discrete ambiguities, with emphasis on linear and nonlinear ambiguities. In Section [V, we give an example of a complete set of eight measurements which resolve all ambiguities, and then present Tables of all such sets of observables. In Section $\nabla$, relations among spin observables are derived using the Fierz identities, which are then used to confirm the complete sets of observables deduced earlier.

We assume that the four Type $\mathcal{S}$ observables are always measured and that the problem is to select the double spin observables which will yield unambiguous total amplitudes. We do not deal with the problem of extracting partial wave amplitudes. 


\section{BILINEAR HELICITY PRODUCTS}

In this section, we introduce the bilinear helicity product (BHP) formulation for discussing spin observables, following the conventions in references [3, 4].

The pseudoscalar meson photoproduction reaction is completely described by four complex helicity amplitudes: $H_{1}, H_{2}, H_{3}$ and $H_{4}$. at each energy and angle. The 16 spin observables, $\Omega^{\alpha}$, consist of the differential cross section $\sigma(\theta)$, plus 3 single and 12 double spin observables. Expressions for these 16 observables in terms of the helicity, transversity and BHP forms are presented in Table [.] All of the 16 observables can be expressed in bilinear helicity product (BHP) form [3]:

$$
\check{\Omega}^{\alpha}=\Omega^{\alpha} \mathcal{I}(\theta)=\frac{1}{2} H_{i}^{*} \Gamma_{i j}^{\alpha} H_{j} \equiv \frac{1}{2}\left\langle H\left|\Gamma^{\alpha}\right| H\right\rangle, \quad \alpha=1, \cdots 16
$$

where summation over repeated indices is implied. We define $\mathcal{I}(\theta)=(k / q) \sigma(\theta)$, where $k$ and $q$ are the momenta of the initial and final states in the center-of-mass frame. The $\check{\Omega}^{\alpha}$ are the "profile function" [3, 4] forms of the spin observables, and $\Gamma^{\alpha}$ matrices are the sixteen $4 \times 4$ Hermitian Gamma matrices. See Appendix A for details about the $\Gamma^{\alpha}$ matrices. The

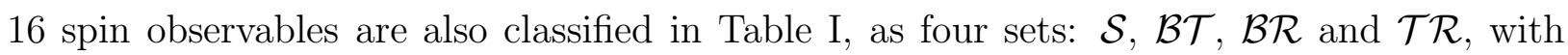
four observables in each set.

A unitary transformation $U^{(4)}$ acting on both the helicity amplitudes and the $\Gamma^{\alpha}$ matrices:

$$
\begin{aligned}
& H_{i} \longrightarrow b_{i}=U_{i j}^{(4)} H_{j} \\
& \Gamma^{\alpha} \longrightarrow \widetilde{\Gamma}^{\alpha}=U^{(4)} \Gamma^{\alpha} U^{\dagger(4)},
\end{aligned}
$$

offers a means of altering amplitudes without changing observables. Such changes in description without changing observables are called canonical transformations, as in mechanics. A particularly useful unitary transformation of this type is the transversity choice [3],

\footnotetext{
${ }^{1}$ These are also often denoted by $S_{1}, N, D$ and $S_{2}$, where $S$ refers to single-flip, $D$ double-flip and $N$ no-flip amplitudes.

${ }^{2}$ For convenience, some of the $\Omega^{\alpha}$ 's in Table [1 were defined with different signs than in Ref. [3].
} 


$$
U^{(4)}=\frac{1}{2}\left(\begin{array}{rrrr}
1 & -i & i & 1 \\
1 & i & -i & 1 \\
1 & i & i & -1 \\
1 & -i & -i & -1
\end{array}\right),
$$

which involves rotating the helicity quantization axis to the direction normal to the scattering plane. The sixteen spin observables can be expressed in this transversity basis by

$$
\check{\Omega}^{\alpha}=\Omega^{\alpha} \mathcal{I}(\theta)=\frac{1}{2} b_{i}^{*} \widetilde{\Gamma}_{i j}^{\alpha} b_{j}=\frac{1}{2}\left\langle b\left|\widetilde{\Gamma}^{\alpha}\right| b\right\rangle, \quad \alpha=1, \cdots 16 .
$$

Note that the corresponding $\widetilde{\Gamma}^{\alpha}$ matrices for the four Type $\mathcal{S}$ measurements are diagonal in the transversity basis, i.e., these observables involve combinations of the squared magnitudes, $\pm\left|b_{i}\right|^{2}$, of the transversity helicity amplitudes. If all four of the Type $\mathcal{S}$ observables are measured (as assumed in this paper), then the double spin observables are used to determine only the phases of the transversity amplitudes. The explicit forms of the Gamma matrices in the transversity basis, $\widetilde{\Gamma}^{\alpha}$, are presented in Appendix A. In this paper, we will work mainly in the transversity basis.

After the above transversity transformation, both the amplitudes and the Gamma matrices are changed, without altering the observables. In the next section, we will introduce unitary transformations corresponding to discrete changes of the amplitudes that can change observables.

This BHP form will be used first to discuss such discrete ambiguities and later for a general approach to the completeness problem.

\section{DISCRETE AMBIGUITIES}

\section{A. General definition of discrete ambiguities}

The extraction of reaction amplitudes from experiments poses an interesting, and sometimes difficult task, because it is a nonlinear problem. To gain insight into the general 
nature of this problem and to define discrete ambiguities broadly, let us consider a reaction described by $N$ complex amplitudes. For pseudoscalar meson photoproduction $N=4$, and we deal with a $4 \times 4$ Gamma algebra. For the general $N$ case one also has a BHP form, but it is represented by a $N \times N$ Clifford algebra. There are $N^{2}$ linearly independent experimental observables which are linear combinations of the $N^{2}$ bilinear products of the $N$ amplitudes. One might assume that $2 N-1$ appropriately chosen observables can determine these $N$ amplitudes, apart from an overall phase factor. However, these $N^{2}$ observables are nonlinearly dependent on each other, and several discrete solutions may satisfy these $2 N-1$ measurements simultaneously. Therefore, more than $2 N-1$ experiments are needed to resolve ambiguities, and the number of additional measurements required is not a fixed number, but depends on the type of measurements already performed [0]. Here we study these discrete ambiguities, following Dean and Lee [6], and find ways to resolve them. Some of the following discussion is equivalent to the methods proposed by Keaton and Workman [2].

In general, the observables (as profile functions) $\left\{\check{\Omega}^{\alpha}\right\}$ can be expressed in a bilinear product form with the $N$ helicity amplitudes $H_{1} \cdots H_{N}$ :

$$
\check{\Omega}^{\alpha}=\Omega^{\alpha} \mathcal{I}(\theta)=\frac{1}{2} H_{i}^{*} \Gamma_{i j}^{\alpha} H_{j}
$$

where $\Gamma^{\alpha}$ are Hermitian $N \times N$ matrices. An ambiguity occurs in extracting the $N$ amplitudes $H_{i}$ from a subset of measurements $\left\{\Omega^{\alpha}\right\} \equiv \Omega^{\alpha_{1}} \cdots \Omega^{\alpha_{M}}$, where $M<N \times N$, when there exists a transformation on the amplitudes $H_{i}$ under which that subset of observables $\left\{\Omega^{\alpha}\right\}$ is invariant. To remove that ambiguity, one need to enlarge the subset $M \rightarrow M+1$ wisely, until no such transformation exists. That defines the process for removing discrete ambiguities.

A trivial case of an ambiguity is an overall phase transformation applied to all $N$ amplitudes $H_{i} \rightarrow e^{i \delta} H_{i}$ with the real $\delta$ independent of $i$. Since $M=N^{2}$, there is no way to remove this ambiguity, which shows that only the relative phases of the amplitudes can be determined. Other nontrivial ambiguities will be discussed later. If the set $\left\{\Omega^{\alpha}\right\}$ of $M$ observables is sufficient to eliminate all ambiguities, then a unique set of amplitudes can be 
extracted. In that case, we call $\left\{\Omega^{\alpha}\right\}$ a complete set of measurements. Linear and nonlinear transformations of the amplitudes can be defined to perform the above test.

As an example of a linear type of transformation that could leave a subset of observables unchanged, consider the following unitary transformation applied to all $N$ helicity amplitudes:

$$
H_{i} \longrightarrow H_{i}^{\prime}=L_{i j} H_{j}
$$

where $L$ is chosen unitary to conserve the differential cross section $\mathcal{I}(\theta)=\frac{1}{2} H_{i}^{*} \delta_{i j} H_{j}=$ $\frac{1}{2} \sum_{i}\left|H_{i}\right|^{2}$. [3 If there exists a unitary $L$ commuting with all $\Gamma^{\alpha}$ 's in the $M<N^{2}$ subset $\Omega^{\alpha_{1}} \cdots \Omega^{\alpha_{M}}$, i.e.,

$$
L^{\dagger} \Gamma^{\alpha_{n}} L=\Gamma^{\alpha_{n}} \quad n=1 \cdots M<N^{2}
$$

then for members of that subset

$$
\check{\Omega}^{\alpha_{n}}=\frac{1}{2} H_{i}^{*} \Gamma_{i j}^{\alpha_{n}} H_{j}=\frac{1}{2} H_{i}^{*}\left(L^{\dagger} \Gamma^{\alpha_{n}} L\right)_{i j} H_{j}=\frac{1}{2} H_{i}^{\prime *} \Gamma_{i j}^{\alpha_{n}} H_{j}^{\prime}
$$

which shows that the subset of observables $\left\{\Omega^{\alpha}\right\}$ are invariant under $L$ and can not be used to distinguish between amplitudes $H_{i}$ and $H_{i}^{\prime}$. Then, there is a linear ambiguity.

Next let us now consider an antilinear transformation acting on all $N$ helicity amplitudes:

$$
H_{i} \longrightarrow H_{i}^{\prime}=A_{i j} H_{j}^{*}
$$

where $A$ is unitary. Any $A$ satisfying

$$
\left(A^{\dagger} \Gamma^{\alpha_{n}} A\right)^{T}=\Gamma^{\alpha_{n}}
$$

where $\alpha_{n}$ corresponds to any observable in the $M<N^{2}$ subset $\Omega^{\alpha_{1}} \cdots \Omega^{\alpha_{M}}$, defines an antilinear ambiguity for $\left\{\Omega^{\alpha_{n}}\right\}$ because

\footnotetext{
${ }^{3}$ Here we suppress density of states factors and use the fact that the cross section is the sum of magnitude-squared helicity amplitudes
} 


$$
\begin{aligned}
\check{\Omega}^{\alpha_{n}} & =\frac{1}{2} H_{i}^{*} \Gamma_{i j}^{\alpha_{n}} H_{j} \\
& =\frac{1}{2} H_{i}^{*}\left(A^{\dagger} \Gamma^{\alpha_{n}} A\right)_{j i} H_{j} \\
& =\frac{1}{2} H_{i}^{*} A_{j k}^{\dagger} \Gamma_{k l}^{\alpha_{n}} A_{l i} H_{j} \\
& =\frac{1}{2}\left(A_{l i} H_{i}^{*}\right) \Gamma_{k l}^{\alpha_{n}}\left(A_{j k}^{\dagger} H_{j}\right) \\
& =\frac{1}{2} H_{k}^{*} \Gamma_{k l}^{\alpha_{n}} H_{l}^{\prime},
\end{aligned}
$$

which shows that members of the measurement subset $\Omega^{\alpha_{n}}$ can not distinguish between amplitudes $H_{i}$ and $H_{i}^{\prime}$.

\section{B. Discrete ambiguities for pseudoscalar meson photoproduction}

For pseudoscalar meson photoproduction $(N=4)$, we have expressed the sixteen spin observables in BHP form using the transversity basis,

$$
\check{\Omega}^{\alpha}=\frac{1}{2} b_{i}^{*} \widetilde{\Gamma}_{i j}^{\alpha} b_{j}
$$

To find associated discrete ambiguities, we need to look for matrices $L$ and $A$ which satisfy Eq. (3.3) and (3.6), respectively. Since the sixteen Hermitian $\widetilde{\Gamma}^{\alpha}$ matrices form a basis for $4 \times 4$ matrices, it is sufficient to find $\widetilde{\Gamma}^{\alpha}$ matrices satisfying Eq. (3.3) and (3.6). Suppose that

we always measure the four Type $\mathcal{S}$ observables: $\check{\Omega}^{1}, \check{\Omega}^{4}, \check{\Omega}^{10}, \check{\Omega}^{12}$. The only $\widetilde{\Gamma}^{\alpha}$ matrices commuting with all four of those $\widetilde{\Gamma}^{\alpha}$ matrices in Type $\mathcal{S}$ are:

$$
L=\widetilde{\Gamma}^{4}, \widetilde{\Gamma}^{10}, \widetilde{\Gamma}^{12} \quad \text { for } \mathcal{S}
$$

(where $L=\widetilde{\Gamma}^{1}$ is not listed because it obviously leaves all amplitudes unchanged). Those $\widetilde{\Gamma}^{\alpha}$ matrices satisfying the antilinear transformation case, Eq. (3.6), are:

$$
A=\widetilde{\Gamma}^{6}, \widetilde{\Gamma}^{8}, \widetilde{\Gamma}^{13}, \widetilde{\Gamma}^{15} \quad \text { for } \mathcal{S}
$$

So $L=\left\{\widetilde{\Gamma}^{4}, \widetilde{\Gamma}^{10}, \widetilde{\Gamma}^{12}\right\}$ are possible candidates for testing for linear ambiguities in any subset of measurements which includes type $\mathcal{S}$ measurements. Similarly, $A=\left\{\widetilde{\Gamma}^{6}, \widetilde{\Gamma}^{8}, \widetilde{\Gamma}^{13}, \widetilde{\Gamma}^{15}\right\}$ 
test for antilinear ambiguities. We believe that all other transformations, assuming type $\mathcal{S}$ measurements, can be constructed from the above basic linear and antilinear unitary transformations.

All sixteen spin observables remain either unchanged or simply change sign under these basic linear and antilinear transformations. The results are given in Table $\mathbb{\text { II }}$ (some of these cases are in Ref. [2]). We are working with transversity amplitudes and correspondingly with the transformed matrices $\widetilde{\Gamma}$. The parallel results are expressed in the helicity basis in Appendix B, wherein the connection to the results of Ref. [2] is made.

If a subset of measured observables are invariant under one of these linear or antilinear transformations, then a discrete ambiguity exists. For example, if we measure $G, F, O_{z}, C_{x}, T_{x}$ and $L_{z}$, in addition to type $\mathcal{S}$, since they are all unchanged under the antilinear transformation with $A=\widetilde{\Gamma}^{6}$, these $4+6=10$ spin observables cannot resolve all ambiguities. N Note that the BDS rule is violated in this case. Therefore, to determine the amplitudes uniquely, one has to choose a set of spin observables that are not all invariant under these $L$ and $A$ transformations. Unfortunately, the above statement provides only necessary but not sufficient conditions to determine unique solutions, since there are also nonlinear ambiguities which are relatively difficult to resolve.

To clarify the above discussion, we note that some transformations of the basic amplitudes leave some set of observables unchanged, while other observables simply change sign. For example, the replacement $b_{3} \rightarrow-b_{3}$, and $b_{4} \rightarrow-b_{4}$, leave the eight observables $\Omega_{1,4,10,12}$ (Type $\mathcal{S}$ ) and the $\Omega_{6,13,8,15}$ (Type $\mathcal{T} \mathcal{R}$ ) unchanged, while the sign of the eight observables $\Omega_{3,5,9,11}($ Type $\mathcal{B} \mathcal{T})$ and $\Omega_{14,7,16,2}($ Type $\mathcal{B R})$ are changed, see Table I. If none of these sign changed observables are among those measured, then we have an ambiguity in determining $b_{3} \& b_{4}$. This particular transformation of the amplitudes can be represented as $b_{i}^{\prime}=U_{i j} b_{i}$ with:

\footnotetext{
${ }^{4}$ In this case, the transformation is $b_{1} \leftrightarrow-b_{2}^{*}$ and $b_{3} \leftrightarrow b_{4}^{*}$, see Eq. (A5).
} 


$$
U=\left(\begin{array}{rrrr}
1 & 0 & 0 & 0 \\
0 & 1 & 0 & 0 \\
0 & 0 & -1 & 0 \\
0 & 0 & 0 & -1
\end{array}\right),
$$

which is identical to $\widetilde{\Gamma}^{4}$. Now consider the effect of such a transformation on all of the observables $\Omega^{\alpha}$. We have:

$$
\check{\Omega}^{\alpha}=\Omega^{\alpha} \mathcal{I}(\theta)=\frac{1}{2} b_{i}^{*} \widetilde{\Gamma}_{i j}^{\alpha} b_{j} \rightarrow \frac{1}{2} b_{i}^{*} U_{k^{\prime} i}^{*} \widetilde{\Gamma}_{k^{\prime} k}^{\alpha} U_{k j} b_{j}, \quad \alpha=1, \cdots 16 .
$$

Since for our particular example $U \rightarrow \widetilde{\Gamma}_{4}$, the effect of this discrete transformation on the transversity amplitudes is equivalent to the following substitution:

$$
\widetilde{\Gamma}^{\alpha} \longrightarrow \widetilde{\Gamma}^{4} \widetilde{\Gamma}^{\alpha} \widetilde{\Gamma}^{4}
$$

The above effect of $\widetilde{\Gamma}^{4}$ on $\widetilde{\Gamma}^{\alpha}$, exactly duplicates the sign changes indicated above that are induced by the $b_{3,4} \rightarrow-b_{3,4}$ substitution. This result is also seen in the third column of Table I and the first column of Table II .

We wish to find a subset of measurements that can be used to deduce a unique set of transversity amplitudes. Once accomplished, the helicity amplitudes can be obtained by the inverse of Eq. (2.4). Two different approaches to this problem are presented: in Section IV, we solve for the phases of the transversity amplitudes directly from spin observables; in Section $\square$, we derive relations between spin observables from the Fierz identities of the $\widetilde{\Gamma}^{\alpha}$ matrices.

\section{COMPLETE SET OF MEASUREMENTS}

Since we assume that we always measure four Type $\mathcal{S}$ observables, the magnitudes of the four transversity amplitudes, $r_{i} \equiv\left|b_{i}\right|$, can always be determined unambiguously. Three double spin observables can in general determine the relative phases between the four helicity amplitudes, but leave us with discrete ambiguities. Therefore, more measurements are required to resolve these ambiguities. We claim the following surprising result: 
In addition to the set $\mathcal{S}$, four appropriately chosen double spin observables are sufficient to determine the amplitudes uniquely.

This means that a total of eight properly chosen measurements can resolve all ambiguities. This result contradicts the BDS rule, which asserted that nine measurements are necessary. In the following discussion, we first provide one explicit example which shows that eight measurements are sufficient. Then we present our complete results and guidelines for all situations.

Here we choose the same measurements as in the example given by BDS [1]. Suppose that we measure $G, F$ and $L_{x}$, along with the set $\mathcal{S}$. We then have the equations (see Column 3 of Table ()

$$
\begin{aligned}
G & =-r_{1} r_{3} \sin \left(\phi_{13}\right)-r_{2} r_{4} \sin \left(\phi_{24}\right) \\
F & =r_{1} r_{3} \sin \left(\phi_{13}\right)-r_{2} r_{4} \sin \left(\phi_{24}\right) \\
L_{x} & =-r_{1} r_{2} \sin \left(\phi_{12}\right)-r_{3} r_{4} \sin \left(\phi_{34}\right),
\end{aligned}
$$

where we write the amplitudes $b_{i}=r_{i} \exp \left(\phi_{i}\right)$ and $\phi_{i j}=\phi_{i}-\phi_{j}$. Except for slightly different conventions, the solutions given by BDS [1] are

$$
\begin{aligned}
& \phi_{13}=\alpha_{13} \quad \text { or } \quad \pi-\alpha_{13} \\
& \phi_{24}=\alpha_{24} \quad \text { or } \quad \pi-\alpha_{24} \\
& \phi_{12}=\beta+\gamma \quad \text { or } \quad \beta+(\pi-\gamma),
\end{aligned}
$$

where $\alpha_{13}, \alpha_{24}, \beta$ and $\gamma$ are defined by ${ }^{5}$

$$
\begin{array}{llrl}
\sin \alpha_{13}=\frac{F-G}{2 r_{1} r_{3}}, & -\frac{\pi}{2} \leq \alpha_{13} \leq \frac{\pi}{2} \\
\sin \alpha_{24}=-\frac{G+F}{2 r_{2} r_{4}}, & -\frac{\pi}{2} \leq \alpha_{24} \leq \frac{\pi}{2}
\end{array}
$$

\footnotetext{
${ }^{5}$ Here $\alpha_{13}$ and $\alpha_{24}$ are uniquely defined. Once $\phi_{13}$ and $\phi_{24}$ (4 choices) are selected, $A$ is fixed and so are $\beta$ and $\gamma$. There are still 2 choices for $\phi_{12}$ (Eq. (4.6)).
} 


$$
\begin{aligned}
\sin \gamma & =-\frac{L_{x}}{A}, \quad-\frac{\pi}{2} \leq \gamma \leq \frac{\pi}{2} \\
\sin \beta & =\frac{r_{3} r_{4} \sin \left(\phi_{13}-\phi_{24}\right)}{A}, \quad \cos \beta=\frac{r_{1} r_{2}+r_{3} r_{4} \cos \left(\phi_{13}-\phi_{24}\right)}{A} \\
A & =\left[r_{1}^{2} r_{2}^{2}+r_{3}^{2} r_{4}^{2}+2 r_{1} r_{2} r_{3} r_{4} \cos \left(\phi_{13}-\phi_{24}\right)\right]^{1 / 2}
\end{aligned}
$$

Therefore, we have an eightfold ambiguity in determining the phases. BDS showed that two more measurements, e.g., $E$ and $L_{z}$, can resolve the ambiguity. But, instead of two, we can show that only one additional appropriately chosen measurement can completely determine the four amplitudes. Instead of the BDS choice of $E$ and $L_{z}$, take just $T_{x}=$ $-r_{1} r_{2} \cos \left(\phi_{12}\right)+r_{3} r_{4} \cos \left(\phi_{34}\right)$ as the fourth double spin observable in addition to $G, F$ and $L_{x}$. Using $L_{x}$ and $T_{x}$ to solve for the $\phi_{12}$ and $\phi_{34}$ phases, we get

$$
\left\{\begin{array} { l } 
{ \phi _ { 1 2 } = - \xi + \alpha _ { 1 2 } } \\
{ \phi _ { 3 4 } = \xi + \alpha _ { 3 4 } }
\end{array} \quad \text { or } \quad \left\{\begin{array}{l}
\phi_{12}=-\xi+\left(\pi-\alpha_{12}\right) \\
\phi_{34}=\xi+\left(\pi-\alpha_{34}\right)
\end{array}\right.\right. \text {, }
$$

where $\alpha_{12}, \alpha_{34}$ and $\xi$ are uniquely determined from experiment by

$$
\begin{aligned}
\sin \alpha_{12} & =-\frac{L_{x}^{2}+T_{x}^{2}+r_{1}^{2} r_{2}^{2}-r_{3}^{2} r_{4}^{2}}{2 r_{1} r_{2} \sqrt{L_{x}^{2}+T_{x}^{2}}}, \quad-\frac{\pi}{2} \leq \alpha_{12} \leq \frac{\pi}{2} \\
\sin \alpha_{34} & =-\frac{L_{x}^{2}+T_{x}^{2}-r_{1}^{2} r_{2}^{2}+r_{3}^{2} r_{4}^{2}}{2 r_{1} r_{2} \sqrt{L_{x}^{2}+T_{x}^{2}}}, \quad-\frac{\pi}{2} \leq \alpha_{34} \leq \frac{\pi}{2} \\
\sin \xi & =\frac{T_{x}}{\sqrt{L_{x}^{2}+T_{x}^{2}}}, \quad \cos \xi=\frac{L_{x}}{\sqrt{L_{x}^{2}+T_{x}^{2}}} .
\end{aligned}
$$

Note that Eq. (4.8) has a twofold ambiguity in determining $\phi_{12}$ and $\phi_{34}$, unlike the fourfold ambiguity for the solutions of $\phi_{13}$ and $\phi_{24}$ (Eq. (4.4) and (4.5)).

Combining the four solutions for $\phi_{13}$ and $\phi_{24}$ (Eq. (4.4) and (4.5)) and the two solutions for $\phi_{12}$ and $\phi_{34}$ (Eq. (4.8)), we now have eight sets of possible solutions. Using the relation $\phi_{34}=\phi_{12}+\phi_{24}-\phi_{13}$, these eight solutions can be expressed by:

$$
2 \xi= \pm\left(\alpha_{12}-\alpha_{34}\right) \pm\left\{\begin{array}{l}
\left(\alpha_{13}-\alpha_{24}\right) \\
\pi-\left(\alpha_{13}+\alpha_{24}\right)
\end{array}\right.
$$

here the two \pm signs are independent. Because all $\alpha$ 's and $\xi$ are fixed, only one of the above eight solutions will hold in general, which tells us that if there is a solution, then it 
is in general a unique solution. Therefore, in this particular case, we have shown that eight spin observables can resolve all ambiguities except the overall phase. All other cases can be evaluated in the same way. We give some guidelines in Appendix $\mathrm{Q}$ and list all the situations (Table [II-VIII) for which eight measurements can completely determine the amplitudes.

\section{RELATIONS FROM FIERZ IDENTITIES}

In the previous section, an elementary, albeit tedious method was used to determine a unique solution. In this section we use a totally different approach to the same problem of determining which set of experiments can determine the four transversity amplitudes without discrete ambiguities.

We know that in field theory [7], bilinear products of currents obey interchange relations known as the Fierz identities. In our problem, we do not deal with the four dimensional space-time, instead we have a four dimensional $\left(b_{1} \cdots b_{4}\right)$ amplitude space. Nevertheless, the properties of the Gamma matrices are characteristic of four dimensional space and thus hermitian versions of the Gamma matrices, along with all of their known properties, are of use to us. Their Fierz identities, which were particularly useful in weak interactions studies, are obtained from the following property of the Gamma matrices:

$$
\Gamma_{i j}^{\alpha} \Gamma_{s t}^{\beta}=C_{\delta \eta}^{\alpha \beta} \Gamma_{i t}^{\delta} \Gamma_{s j}^{\eta}
$$

where $C_{\delta \eta}^{\alpha \beta} \equiv \frac{1}{16} \operatorname{Tr}\left(\Gamma^{\delta} \Gamma^{\alpha} \Gamma^{\eta} \Gamma^{\beta}\right)$. These identities are properties of the (hermitian) Gamma matrices as discussed in Appendix A. We can therefore use the above Fierz identities for the Gamma matrices, even though we are in a context entirely different from their field theory origin.

Applying the Fierz transformations to the BHP forms for spin observables, yields the following set of relations between observables:

$$
\begin{aligned}
\check{\Omega}^{\alpha} \check{\Omega}^{\beta} & =\left(\frac{1}{2} b_{i}^{*} \widetilde{\Gamma}_{i j}^{\alpha} b_{j}\right)\left(\frac{1}{2} b_{s}^{*} \widetilde{\Gamma}_{s t}^{\beta} b_{t}\right) \\
& =C_{\delta \eta}^{\alpha \beta}\left(\frac{1}{2} b_{i}^{*} \widetilde{\Gamma}_{i t}^{\delta} b_{t}\right)\left(\frac{1}{2} b_{s}^{*} \widetilde{\Gamma}_{s j}^{\delta} b_{j}\right)
\end{aligned}
$$




$$
=C_{\delta \eta}^{\alpha \beta} \check{\Omega}^{\delta} \check{\Omega}^{\eta}
$$

or, since the above profile functions satisfy $\check{\Omega}^{\alpha} \equiv \Omega^{\alpha} \mathcal{I}$, for all $\alpha$ :

$$
\Omega^{\alpha} \Omega^{\beta}=C_{\delta \eta}^{\alpha \beta} \Omega^{\delta} \Omega^{\eta}
$$

All distinct Fierz relations derived from Eq. (5.3) are presented in Appendix D. In the rest of this section, we will show that these relations provide an alternate way to obtain some useful results.

\section{A. Fierz observable constraints and bounds}

The Fierz relations yield explicit and rigorous relationships between observables. Of course, such relationships can be derived from the bilinear structure of the observables, with much effort. That effort is now replaced by simply invoking the well-known Fierz rules as a general property. That allows us to avoid much algebra and to find all relations in one step. There are direct physical consequences of these relations.

For example, from Eq. (L.tr), (L.br) and (L.bt) in Appendix D, it can be seen that if three double spin observables in a type set are known, then the fourth member of that type is uniquely determined. The fourth measurement is thus redundant.

The Fierz relations can also be used to derive bounds on measurements. For example, from Eq. (L.tr) and (S.tr),

$$
\begin{aligned}
& \left(\Omega_{6}\right)^{2}+\left(\Omega_{13}\right)^{2}+\left(\Omega_{8}\right)^{2}+\left(\Omega_{15}\right)^{2} \pm 2\left(\Omega_{6} \Omega_{15}-\Omega_{8} \Omega_{13}\right) \\
= & \left(\Omega_{1}\right)^{2}+\left(\Omega_{4}\right)^{2}-\left(\Omega_{10}\right)^{2}-\left(\Omega_{12}\right)^{2} \pm 2\left(\Omega_{1} \Omega_{4}-\Omega_{10} \Omega_{12}\right)
\end{aligned}
$$

we obtain

$$
\left(\Omega_{6} \pm \Omega_{15}\right)^{2}+\left(\Omega_{8} \mp \Omega_{13}\right)^{2}=\left(\Omega_{1} \pm \Omega_{4}\right)^{2}-\left(\Omega_{10} \pm \Omega_{12}\right)^{2} .
$$

The left hand side of the equation is positive, so is the right hand side. Therefore, Eq. (5.5) gives a bound relation 


$$
\Omega_{1} \pm \Omega_{4} \geq\left|\Omega_{10} \pm \Omega_{12}\right| \quad \text { or } \quad 1 \pm \Sigma \geq|T \pm P|
$$

Other bounds, within the set $\mathcal{S}$, can be derived in the same way:

$$
1 \pm T \geq|P \pm \Sigma|, \quad 1 \pm P \geq|\Sigma \pm T|
$$

Since the left hand sides of Eqs. (S.bt), (S.br) and (S.tr) in Appendix D are positive, we can deduce the bounds

$$
\begin{aligned}
& 1+\Sigma^{2} \geq P^{2}+T^{2} \\
& 1+T^{2} \geq \Sigma^{2}+P^{2} \\
& 1+P^{2} \geq \Sigma^{2}+T^{2}
\end{aligned}
$$

as well as $P^{2} \leq 1, \Sigma^{2} \leq 1$ and $T^{2} \leq 1$

In this way all bounds among spin observables given by BDS [1] and Goldstein et al. [8] can be obtained using these Fierz relations.

\section{B. Fierz and selection of experiments}

We determined how to pick a complete set of measurements by solving trigonometric equations in Section [V]. Here we show that the Fierz relations can accomplish the same task.

Take the case of the four double spin observables $G, H, O_{x}$ and $C_{x}\left(\Omega_{3,5,14,16}\right)$. From Eq. (S.bt), we can determine $\left(\Omega_{9}\right)^{2}+\left(\Omega_{11}\right)^{2}$. Therefore, Eq. (S.br) and (S.b) can yield the magnitudes of $\Omega_{2}$ and $\Omega_{7}$. Finally, invoking Eq. (L.br) we can uniquely decide $\Omega_{2}$ and $\Omega_{7}$. By selecting the appropriate equations from Appendix $\mathbb{D}$, one can determine all other

observables. Once all the observables $\check{\Omega}_{\alpha}$ are known, we can use $\frac{1}{2} b_{i}^{*} b_{j}=\sum_{\alpha} \widetilde{\Gamma}_{i, j}^{\alpha} \check{\Omega}_{\alpha}$, or $\frac{1}{2} H_{i}^{*} H_{j}=\sum_{\alpha} \Gamma_{i, j}^{\alpha} \check{\Omega}_{\alpha}$, to obtain the amplitudes; here the sum is over $\alpha=1 \cdots 16$ - all the now known observables.

In this specific case, we show that the chosen eight measurements resolve the ambiguities. All other cases can be examined in similar way using properly selected Fierz relations to 
determine the unmeasured observables, although sometimes it becomes rather awkward to find the right set of Fierz relations needed for the task. The same result that was found earlier, which is summarized in Tables III-VIII, are recovered by this second method. One advantage of the Fierz-based method is that it provides a procedure that could possibly be generalized to reactions with $N>4$ amplitudes.

\section{CONCLUSIONS}

We have re-examined the classic question of how many observables are required in pseudoscalar meson photoproduction to completely and unambiguously extract the basic amplitudes from experiment. The four magnitudes and three phases suggest that, aside from an overall arbitrary phase, only seven experiments are needed. However, seven experiments do not suffice to resolve discrete ambiguities, as has been discussed, most recently by Keaton and Workman. Stimulated by that observation, we have investigated the question of the number of spin observables needed to determine the transversity amplitudes (assuming the cross section plus all single spin observables are measured). It is convenient to transform to transversity amplitudes, which use the normal to the scattering plane as the quantization axis. In that case, the cross section plus the three single spin observables determine the magnitudes of the transversity amplitudes, while the double spin observables play the role of determining their phases. It is found that by carefully selecting four of the double spin observables it is possible to extract all of the requisite phases without discrete ambiguities.

This is illustrated following the same procedure used in the classic BDS paper, by explicitly expressing observables in terms of the magnitudes and relative phases of the amplitudes. As an alternate approach, we expressed all observables in terms of bilinear helicity product forms, which maps the algebra of observables to the algebra of Hermitian versions of the well known $4 \times 4$ Gamma matrices. This mapping allows us to make unitary (canonical) transformations which have no effect on the observables, as illustrated by the transformation to the transversity basis. In addition, there are transformations which can store the discrete 
ambiguities. By finding the set of those unitary matrices that describe the discrete transformations, it is possible to delineate which experiments resolve such discrete ambiguities.

In addition, once it is recognized that the algebra of observables maps over to the algebra of the $4 \times 4$ Gamma matrices, we can use all known properties of the $4 \times 4$ matrices. In our case, the four dimensional space is not that of space-time, but is rather the four dimensional helicity space. Nevertheless, the Gamma matrices have the well known properties of four dimensional space. One property that is particularly interesting is the Fierz transformation. It is shown that the Fierz transformation properties lead to relationships between observables which can be used to provide constraints and inequalities rules for observables.

The Fierz transformation can also be used as an alternate way to prove that a set of

eight experiments can be selected to form a complete set of measurements. All examples of the 4 sets of double spin observables are presented in Table form, since we have not been able to express this result using a simple guideline.

\section{ACKNOWLEDGMENTS}

The authors wish to thank Dr. R. Workman for his comments, which stimulated us to examine this problem. We also wish to thank Dr. Bijan Saghai for helpful discussions.

\section{APPENDIX A: GAMMA MATRICES}

The sixteen $4 \times 4 \Gamma$ matrices are Hermitian versions of the familiar Dirac matrices:

$$
\Gamma^{\alpha=1 \cdots 16}=1, \gamma^{0}, i \vec{\gamma}, i \sigma^{0 x}, i \sigma^{0 y}, i \sigma^{0 z}, i \sigma^{x y}, i \sigma^{x z}, i \sigma^{z y}, i \gamma^{5} \gamma^{0}, \gamma^{5} \vec{\gamma}, \gamma^{5}
$$

They have the following properties which are used in this paper:

(a) $\Gamma^{\alpha}$ are Hermitian and unitary.

(b) $\operatorname{Tr}\left(\Gamma^{\alpha} \Gamma^{\beta}\right)=4 \delta_{\alpha \beta}$. 
(c) $\Gamma^{\alpha}$ are linearly independent. Therefore, they form a complete set (a basis) for $4 \times 4$ matrices. Using Property (b), any $4 \times 4$ matrices $X$ can be expanded as $X=\sum_{\alpha} C_{\alpha} \Gamma^{\alpha}$ with $C_{\alpha}=\frac{1}{4} \operatorname{Tr}\left(\Gamma^{\alpha} X\right)$.

(d) $\sum_{\alpha} \Gamma_{b a}^{\alpha} \Gamma_{s t}^{\alpha}=4 \delta_{a s} \delta_{b t}$.

(e) $\Gamma^{\alpha} \Gamma^{\beta}=\rho_{\alpha \beta \gamma} \Gamma^{\gamma}$ with $\rho_{\alpha \beta \gamma}=\frac{1}{4} \operatorname{Tr}\left(\Gamma^{\alpha} \Gamma^{\beta} \Gamma^{\gamma}\right)$.

(f) $\frac{1}{4} \rho_{\alpha \gamma \delta} \rho_{\beta \gamma \eta}=\frac{1}{16} \operatorname{Tr}\left(\Gamma^{\delta} \Gamma^{\alpha} \Gamma^{\eta} \Gamma^{\beta}\right) \equiv C_{\delta \eta}^{\alpha \beta}$, which is used for the Fierz transformation in Section V.

These properties are preserved under any unitary transformation, e.g., the transversity transformation $U^{(4)}$ (defined in Eq. (2.4)). Therefore, the $\widetilde{\Gamma}$ matrices in the transversity basis have the same properties as the original $\Gamma$ matrices. These sixteen $\widetilde{\Gamma}$ matrices can be grouped into four classes with four members in each class according to their "shape." (By shape, we mean the location of nonzero entries in $\widetilde{\Gamma}$ matrices.) The four shapes are: diagonal $(D)$; right parallelogram $(P R)$; antidiagonal $(A D)$; and left parallelogram $(P L)$ [3]. In the transversity basis, these four shape classes correspond to $\mathcal{S}, \mathcal{B T}, \mathcal{B R}$ and $\mathcal{T} \mathcal{R}$ type experiments. Here, we give their explicit expressions:

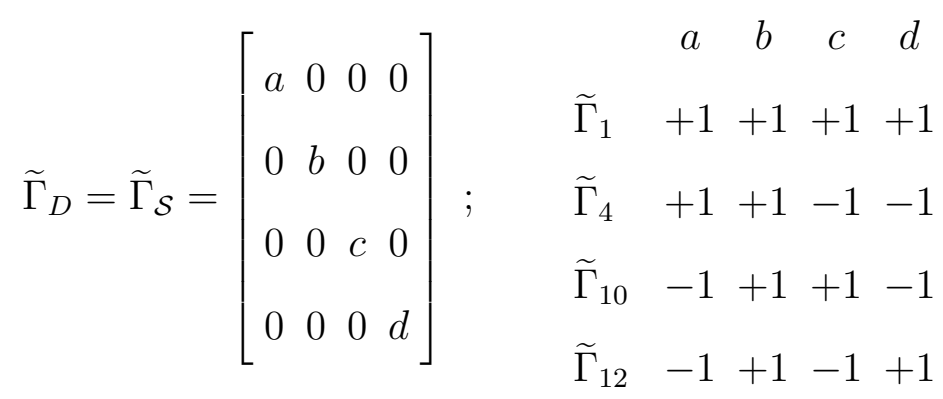

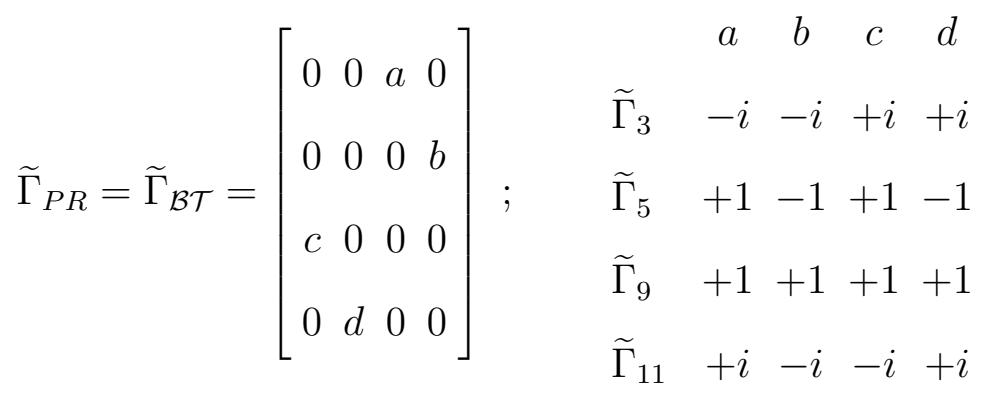




$$
\begin{aligned}
& \widetilde{\Gamma}_{A D}=\widetilde{\Gamma}_{\mathcal{B R}}=\left[\begin{array}{cccc}
0 & 0 & 0 & a \\
0 & 0 & b & 0 \\
0 & c & 0 & 0 \\
d & 0 & 0 & 0
\end{array}\right] ; \quad \begin{array}{cccccc} 
& & a & b & c & d \\
& \widetilde{\Gamma}_{14} & -1 & +1 & +1 & -1 \\
\widetilde{\Gamma}_{7} & -i & -i & +i & +i \\
& \widetilde{\Gamma}_{16} & +i & -i & +i & -i \\
& \widetilde{\Gamma}_{2} & +1 & +1+1 & +1
\end{array} \\
& \widetilde{\Gamma}_{P L}=\widetilde{\Gamma}_{\mathcal{T} \mathcal{R}}=\left[\begin{array}{cccc}
0 & a & 0 & 0 \\
b & 0 & 0 & 0 \\
0 & 0 & 0 & c \\
0 & 0 & d & 0
\end{array}\right] ; \quad \begin{array}{ccccc} 
& a & b & c & d \\
\widetilde{\Gamma}_{6} & -1 & -1 & +1 & +1 \\
\widetilde{\Gamma}_{13} & +i & -i & -i & +i \\
\widetilde{\Gamma}_{8} & -i & +i & -i & +i \\
\widetilde{\Gamma}_{15} & -1 & -1 & -1 & -1
\end{array}
\end{aligned}
$$

\section{APPENDIX B: DISCRETE AMBIGUITIES IN HELICITY BASIS}

In Ref. [2], KW gave discrete ambiguity relations associated with transformations of helicity amplitudes:

Ambiguity I

$$
H_{1} \longleftrightarrow \begin{aligned}
& H_{4} \\
& H_{2}
\end{aligned} \text {; i.e. }\left[\begin{array}{l}
H_{1} \\
H_{2} \\
H_{3} \\
H_{4}
\end{array}\right] \longrightarrow\left[\begin{array}{c}
H_{1}^{\prime} \\
H_{2}^{\prime} \\
H_{3}^{\prime} \\
H_{4}^{\prime}
\end{array}\right]=\left[\begin{array}{cccc}
0 & 0 & 0 & +1 \\
0 & 0 & -1 & 0 \\
0 & -1 & 0 & 0 \\
+1 & 0 & 0 & 0
\end{array}\right]\left[\begin{array}{l}
H_{1} \\
H_{2} \\
H_{3} \\
H_{4}
\end{array}\right]=\Gamma^{4}\left[\begin{array}{c}
H_{1} \\
H_{2} \\
H_{3} \\
H_{4}
\end{array}\right] .
$$

Ambiguity II

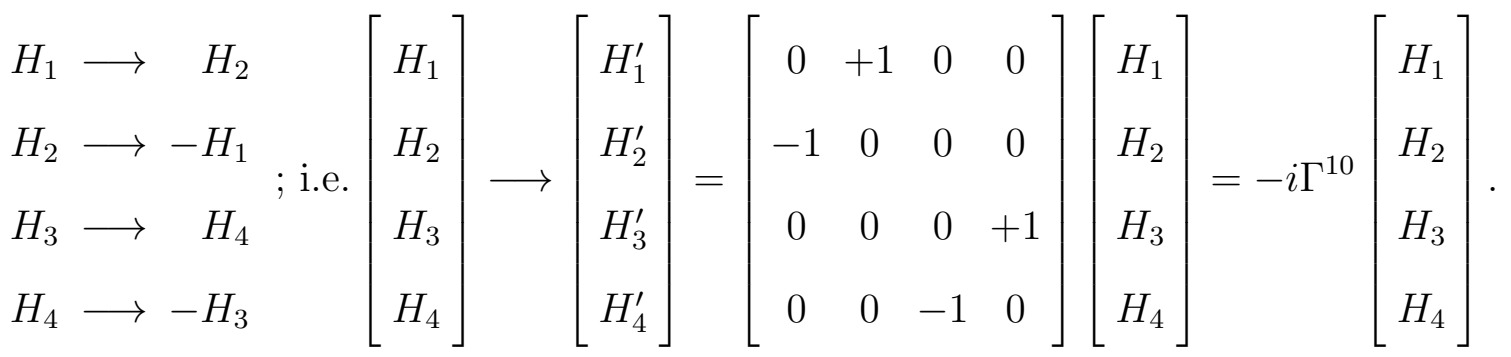

Ambiguity III 


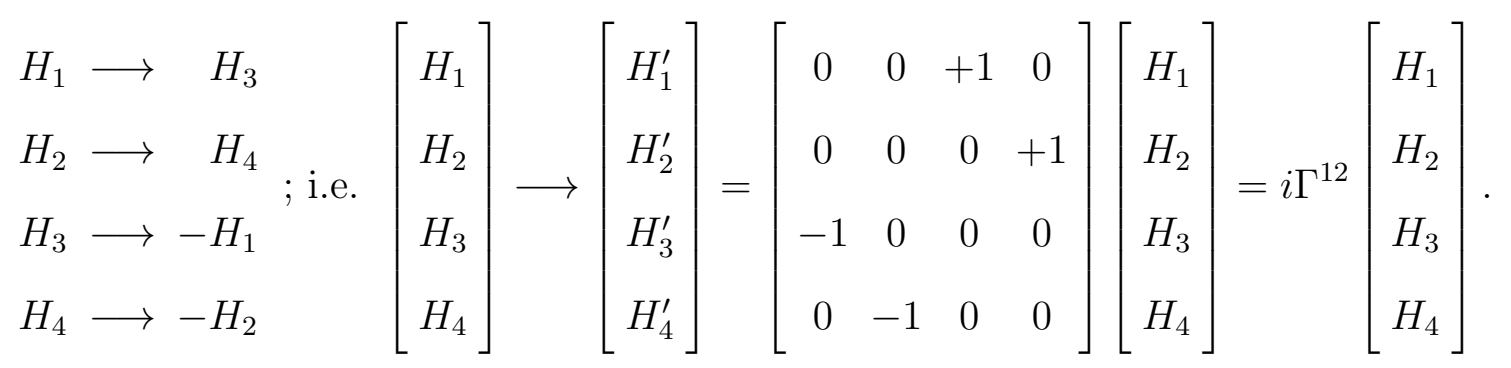

Ambiguity IV

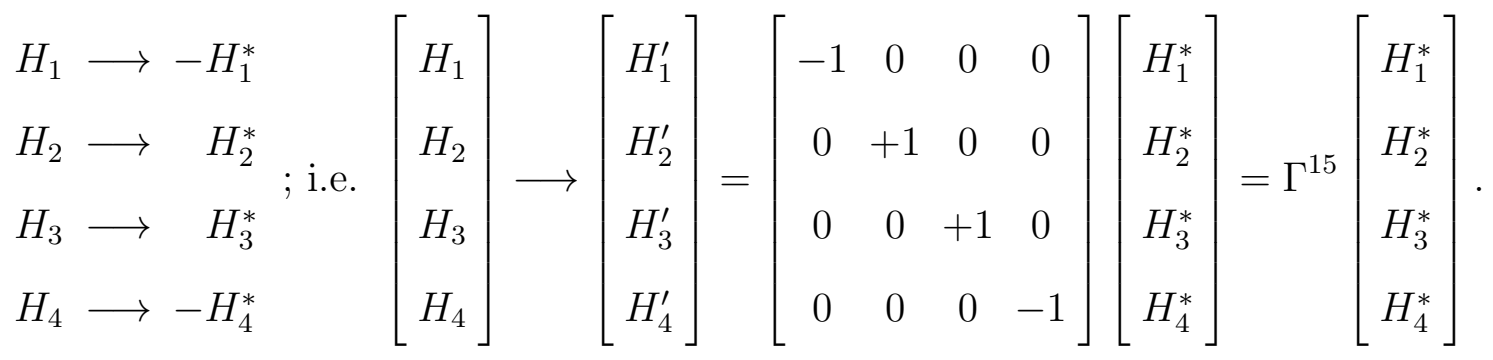

Note that in the above the helicity amplitudes and Gamma matrices are in the original basis. Since we work exclusively in the transversity basis in this paper, it is convenient to express the above ambiguities in the transversity basis:

$$
\begin{gathered}
{\left[\begin{array}{l}
b_{1} \\
b_{2} \\
b_{3} \\
b_{4}
\end{array}\right] \longrightarrow\left[\begin{array}{l}
b_{1}^{\prime} \\
b_{2}^{\prime} \\
b_{3}^{\prime} \\
b_{4}^{\prime}
\end{array}\right]=\left[\begin{array}{cccc}
+1 & 0 & 0 & 0 \\
0 & +1 & 0 & 0 \\
0 & 0 & -1 & 0 \\
0 & 0 & 0 & -1
\end{array}\right]\left[\begin{array}{l}
b_{1} \\
b_{2} \\
b_{3} \\
b_{4}
\end{array}\right]=\widetilde{\Gamma}^{4}\left[\begin{array}{l}
b_{1} \\
b_{2} \\
b_{3} \\
b_{4}
\end{array}\right] .} \\
{\left[\begin{array}{l}
b_{1} \\
b_{2} \\
b_{3} \\
b_{4}
\end{array}\right] \longrightarrow\left[\begin{array}{l}
b_{1}^{\prime} \\
b_{2}^{\prime} \\
b_{3}^{\prime} \\
b_{4}^{\prime}
\end{array}\right]=\left[\begin{array}{cccc}
-1 & 0 & 0 & 0 \\
0 & +1 & 0 & 0 \\
0 & 0 & +1 & 0 \\
0 & 0 & 0 & -1
\end{array}\right]\left[\begin{array}{l}
b_{1} \\
b_{2} \\
b_{3} \\
b_{4}
\end{array}\right]=-i \widetilde{\Gamma}^{10}\left[\begin{array}{l}
b_{1} \\
b_{2} \\
b_{3} \\
b_{4}
\end{array}\right] .} \\
{\left[\begin{array}{l}
b_{1} \\
b_{2} \\
b_{3} \\
b_{4}
\end{array}\right] \longrightarrow\left[\begin{array}{l}
\text { Ambiguity III } \\
b_{1}^{\prime} \\
b_{2}^{\prime} \\
b_{3}^{\prime} \\
b_{4}^{\prime}
\end{array}\right]=\left[\begin{array}{cccc}
-1 & 0 & 0 & 0 \\
0 & +1 & 0 & 0 \\
0 & 0 & -1 & 0 \\
0 & 0 & 0 & +1
\end{array}\right]\left[\begin{array}{l}
b_{1} \\
b_{2} \\
b_{3} \\
b_{4}
\end{array}\right]=i \widetilde{\Gamma}^{12}\left[\begin{array}{l}
b_{1} \\
b_{2} \\
b_{3} \\
b_{4}
\end{array}\right] .}
\end{gathered}
$$




$$
\left[\begin{array}{l}
b_{1} \\
b_{2} \\
b_{3} \\
b_{4}
\end{array}\right] \longrightarrow\left[\begin{array}{c}
b_{1}^{\prime} \\
b_{2}^{\prime} \\
b_{3}^{\prime} \\
b_{4}^{\prime}
\end{array}\right]=\left[\begin{array}{cccc}
0 & -1 & 0 & 0 \\
-1 & 0 & 0 & 0 \\
0 & 0 & 0 & -1 \\
0 & 0 & -1 & 0
\end{array}\right]\left[\begin{array}{c}
b_{1}^{*} \\
b_{2}^{*} \\
b_{3}^{*} \\
b_{4}^{*}
\end{array}\right]=\widetilde{\Gamma}^{15}\left[\begin{array}{c}
b_{1}^{*} \\
b_{2}^{*} \\
b_{3}^{*} \\
b_{4}^{*}
\end{array}\right] .
$$

Ambiguity I, II and III are equivalent to our linear ambiguity $L=\widetilde{\Gamma}^{4}, \widetilde{\Gamma}^{10}$, and $\widetilde{\Gamma}^{12}$ except for irrelevant phases (see Table 【I). Ambiguity IV corresponds to our antilinear ambiguity $A=\widetilde{\Gamma}^{15}$. And the other three antilinear ambiguities in Table 피, $A=\widetilde{\Gamma}^{6}, \widetilde{\Gamma}^{13}$, and $\widetilde{\Gamma}^{8}$, can be constructed by Ambiguity IV and the three linear ambiguity (Ambiguity I to III). It is shown explicitly by

$$
\begin{aligned}
\widetilde{\Gamma}^{6} & =\widetilde{\Gamma}^{4} \widetilde{\Gamma}^{15} \\
\widetilde{\Gamma}^{13} & =i \widetilde{\Gamma}^{10} \widetilde{\Gamma}^{15} \\
\widetilde{\Gamma}^{8} & =-i \widetilde{\Gamma}^{12} \widetilde{\Gamma}^{15} .
\end{aligned}
$$

Here we recover the results given by KW (Ref. [2]).

\section{APPENDIX C: COMPLETE SETS OF EIGHT MEASUREMENTS}

Here we give rules for choosing four double spin observables which can resolve the ambiguities when they are taken together with $\sigma(\theta), \Sigma, T$ and $P$. These rules are not expressed succinctly, and we can not yet provide simple physical guidance. Some may find Table IIIVIII also useful for choosing the appropriate measurements.

Define $\mathcal{A}, \mathcal{B}, \mathcal{C}, \mathcal{D}, \mathcal{E}$ and $\mathcal{F}$ as sets of pairs of double spin observables:

$$
\begin{array}{r}
\left\{(H, E),\left(O_{x}, C_{z}\right),\left(T_{x}, L_{z}\right)\right\}=\mathcal{A} \\
\left\{(G, F),\left(O_{z}, C_{x}\right),\left(T_{z}, L_{x}\right)\right\}=\mathcal{B} \\
\left\{(H, F),\left(O_{x}, C_{x}\right),\left(T_{x}, T_{z}\right)\right\}=\mathcal{C} \\
\left\{(G, H),\left(O_{x}, O_{z}\right),\left(T_{x}, L_{x}\right)\right\}=\mathcal{D}
\end{array}
$$




$$
\begin{gathered}
\left\{(E, F),\left(C_{x}, C_{z}\right),\left(T_{z}, L_{z}\right)\right\}=\mathcal{E} \\
\left\{(G, E),\left(O_{z}, C_{z}\right),\left(L_{x}, L_{z}\right)\right\}=\mathcal{F},
\end{gathered}
$$

and $\mathcal{X}$ and $\mathcal{Y}$ as sets of double spin observables:

$$
\begin{aligned}
& \left\{H, E, O_{x}, C_{z}, T_{x}, L_{z}\right\}=\mathcal{X} \\
& \left\{G, F, O_{z}, C_{x}, T_{z}, L_{x}\right\}=\mathcal{Y} .
\end{aligned}
$$

There are four situations in choosing four double spin observables:

(a) $\underline{2+2}$ cases: Pick one pair of double spin observables from the same type $(\mathcal{B T}, \mathcal{B R}$ or $\mathcal{T} \mathcal{R}$ ), and another pair from another type. Here are the $2+2$ cases which can determine the amplitudes uniquely:

1. $2 \mathcal{B} \mathcal{T}+2 \mathcal{T} \mathcal{R}$ cases: At least one pair belongs to set $\mathcal{D}$ or $\mathcal{E}$, i.e., at least one pair is $(G, H),(E, F),\left(T_{x}, L_{x}\right)$ or $\left(T_{z}, L_{z}\right)$.

2. $2 \mathcal{B} \mathcal{T}+2 \mathcal{B R}$ cases: At least one pair belongs to set $\mathcal{C}$ or $\mathcal{F}$, i.e., at least one pair is $(G, E),(H, F),\left(O_{x}, C_{x}\right)$ or $\left(O_{z}, C_{z}\right)$.

3. $2 \mathcal{B R}+2 \mathcal{T} \mathcal{R}$ cases: The $\mathcal{B} \mathcal{R}$ pair belongs to set $\mathcal{D}$ or $\mathcal{E}$, or the $\mathcal{T} \mathcal{R}$ pair belongs to set $\mathcal{C}$ or $\mathcal{F}$, i.e., at least one pair is $\left(O_{x}, O_{z}\right),\left(C_{x}, C_{z}\right),\left(T_{x}, T_{z}\right)$ or $\left(L_{x}, L_{z}\right)$.

(b) $\underline{2+1+1}$ cases: Pick one pair of double spin observables from one type of $\mathcal{B} \mathcal{T}, \mathcal{B R}$ or $\mathcal{T} \mathcal{R}$, and one observable from each of the remaining two types. The followings are the only $2+1+1$ situations under which the ambiguities are not resolved:

1. When the pair belongs to set $\mathcal{A}$ and the other two observables belong to the same set of $\mathcal{X}$ or $\mathcal{Y}$.

2. When the pair belongs to set $\mathcal{B}$ and the other two observables belong to the different set of $\mathcal{X}$ and $\mathcal{Y}$.

(c) $\underline{3+1}$ cases: Pick 3 double spin observables from one type and one observable from other types. Ambiguities can not be resolved in these cases. 
(d) $\underline{4}$ cases: Pick all 4 double spin observables from the same type. They can never determine the amplitudes uniquely.

\section{APPENDIX D: FIERZ RELATIONS}

In this appendix, we display all of the Fierz relations as contained in Eq. (5.3). We select

values for the index $\alpha \& \beta$ and then evaluate the coefficient $C_{\delta \eta}^{\alpha \beta}$, from the trace rules in Appendix A. There are $16 \times 16=256$ choices for the pair $\alpha, \beta$; however, due to symmetries and the fact that many of the resulting equations are redundant, we can reduce the Fierz results to the following 37 equations. There continue to be some redundancy in these, but that is hard to judge since they are nonlinear equations.

The 37 surviving equations that are obtained using the Fierz identities are organized according to the following scheme:

a. Relations (L.tr) and (S.tr) involve only $\mathcal{S}$ and $\mathcal{T} \mathcal{R}$ types, etc.;

b. Relations (Q.b) and (S.b) involve only $\mathcal{B} \mathcal{T}$ and $\mathcal{B R}$ types, etc.;

c. Relations (Q.bt.1-4), (Q.br.1-4), (Q.tr.1-4) and (L.1-12) involve all four $\mathcal{T} \mathcal{R}, \mathcal{B} \mathcal{T}$, $\mathcal{B R}$, and $\mathcal{S}$ types.

The label "L" is used for Linear (LHS) to Quadratic (RHS) relations (with $\alpha=1$ selected). The label "Q" denotes purely quadratic relations on right and left sides, while

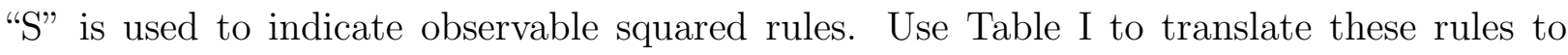
the notation $(\sigma, \Sigma, T, P) \mathcal{S} ;(G, H, E, F) \mathcal{B} \mathcal{T} ;\left(O_{x}, O_{z}, C_{x}, C_{z}\right) \mathcal{B R} ;\left(T_{x}, T_{z}, L_{x}, L_{z}\right) \mathcal{T} \mathcal{R}$. Note that these relations hold true at all energies and all angles. The type $\mathcal{S}$ observables $\Omega_{1,4,10,12}$ are all assumed to be known.

The following relations have been organized to help in seeking connections between observables, which is important in showing that not all observables need to be measured. They have interesting patterns which might help in examining questions of interdependence between observables and in future generalizations to reactions with $N>4$ amplitudes.

Linear-Quadratic Relations: 


$$
\begin{aligned}
& \Omega_{1}=1=\frac{1}{4} \sum_{\alpha=1}^{16}\left(\Omega_{\alpha}\right)^{2} \\
& \Omega_{4}=\Omega_{10} \Omega_{12}+\Omega_{6} \Omega_{15}-\Omega_{8} \Omega_{13} \\
& \Omega_{10}=\Omega_{4} \Omega_{12}+\Omega_{2} \Omega_{14}+\Omega_{7} \Omega_{16} \\
& \Omega_{12}=\Omega_{4} \Omega_{10}+\Omega_{3} \Omega_{11}-\Omega_{5} \Omega_{9}
\end{aligned}
$$

$$
\begin{aligned}
& \Omega_{3}=+\Omega_{11} \Omega_{12}-\Omega_{7} \Omega_{15}+\Omega_{14} \Omega_{8} \\
& \Omega_{5}=-\Omega_{9} \Omega_{12}+\Omega_{7} \Omega_{13}-\Omega_{14} \Omega_{6} \\
& \Omega_{9}=-\Omega_{5} \Omega_{12}-\Omega_{2} \Omega_{15}-\Omega_{16} \Omega_{8} \\
& \Omega_{11}=+\Omega_{3} \Omega_{12}+\Omega_{2} \Omega_{13}+\Omega_{16} \Omega_{6}
\end{aligned}
$$

$$
\Omega_{2}=\Omega_{14} \Omega_{10}-\Omega_{9} \Omega_{15}+\Omega_{11} \Omega_{13}
$$

Quadratic Relations:

$$
\begin{aligned}
& \Omega_{2} \Omega_{7}-\Omega_{14} \Omega_{16}-\Omega_{3} \Omega_{9}-\Omega_{5} \Omega_{11}=0 \\
& \Omega_{3} \Omega_{5}+\Omega_{9} \Omega_{11}+\Omega_{6} \Omega_{8}+\Omega_{13} \Omega_{15}=0 \\
& \Omega_{2} \Omega_{16}-\Omega_{7} \Omega_{14}-\Omega_{6} \Omega_{13}-\Omega_{8} \Omega_{15}=0
\end{aligned}
$$




$$
\begin{aligned}
& \Omega_{4} \Omega_{3}-\Omega_{10} \Omega_{11}+\Omega_{7} \Omega_{6}+\Omega_{14} \Omega_{13}=0 \\
& \Omega_{4} \Omega_{5}+\Omega_{10} \Omega_{9}+\Omega_{7} \Omega_{8}+\Omega_{14} \Omega_{15}=0 \\
& \Omega_{4} \Omega_{9}+\Omega_{10} \Omega_{5}+\Omega_{2} \Omega_{6}-\Omega_{16} \Omega_{13}=0 \\
& \Omega_{4} \Omega_{11}-\Omega_{10} \Omega_{3}+\Omega_{2} \Omega_{8}-\Omega_{16} \Omega_{15}=0
\end{aligned}
$$

$$
\begin{aligned}
& \Omega_{4} \Omega_{14}-\Omega_{12} \Omega_{2}+\Omega_{3} \Omega_{13}+\Omega_{5} \Omega_{15}=0 \\
& \Omega_{4} \Omega_{7}-\Omega_{12} \Omega_{16}+\Omega_{3} \Omega_{6}+\Omega_{5} \Omega_{8}=0 \\
& \Omega_{4} \Omega_{16}-\Omega_{12} \Omega_{7}-\Omega_{9} \Omega_{13}-\Omega_{11} \Omega_{15}=0 \\
& \Omega_{4} \Omega_{2}-\Omega_{12} \Omega_{14}+\Omega_{9} \Omega_{6}+\Omega_{11} \Omega_{8}=0
\end{aligned}
$$

$$
\begin{aligned}
& \Omega_{10} \Omega_{6}-\Omega_{12} \Omega_{15}+\Omega_{5} \Omega_{2}-\Omega_{11} \Omega_{7}=0 \\
& \Omega_{10} \Omega_{13}+\Omega_{12} \Omega_{8}-\Omega_{5} \Omega_{16}-\Omega_{11} \Omega_{14}=0 \\
& \Omega_{10} \Omega_{8}+\Omega_{12} \Omega_{13}-\Omega_{3} \Omega_{2}+\Omega_{9} \Omega_{7}=0 \\
& \Omega_{10} \Omega_{15}-\Omega_{12} \Omega_{6}+\Omega_{3} \Omega_{16}+\Omega_{9} \Omega_{14}=0
\end{aligned}
$$

Square Relations:

$$
\begin{gathered}
\left(\Omega_{3}\right)^{2}+\left(\Omega_{5}\right)^{2}+\left(\Omega_{9}\right)^{2}+\left(\Omega_{11}\right)^{2}=\left(\Omega_{1}\right)^{2}-\left(\Omega_{4}\right)^{2}-\left(\Omega_{10}\right)^{2}+\left(\Omega_{12}\right)^{2} \\
\left(\Omega_{14}\right)^{2}+\left(\Omega_{7}\right)^{2}+\left(\Omega_{16}\right)^{2}+\left(\Omega_{2}\right)^{2}=\left(\Omega_{1}\right)^{2}-\left(\Omega_{4}\right)^{2}+\left(\Omega_{10}\right)^{2}-\left(\Omega_{12}\right)^{2} \\
\left(\Omega_{6}\right)^{2}+\left(\Omega_{13}\right)^{2}+\left(\Omega_{8}\right)^{2}+\left(\Omega_{15}\right)^{2}=\left(\Omega_{1}\right)^{2}+\left(\Omega_{4}\right)^{2}-\left(\Omega_{10}\right)^{2}-\left(\Omega_{12}\right)^{2} \\
\left(\Omega_{3}\right)^{2}+\left(\Omega_{5}\right)^{2}-\left(\Omega_{9}\right)^{2}-\left(\Omega_{11}\right)^{2}=\left(\Omega_{14}\right)^{2}+\left(\Omega_{7}\right)^{2}-\left(\Omega_{16}\right)^{2}-\left(\Omega_{2}\right)^{2} \\
-\left(\Omega_{3}\right)^{2}+\left(\Omega_{5}\right)^{2}-\left(\Omega_{9}\right)^{2}+\left(\Omega_{11}\right)^{2}=\left(\Omega_{6}\right)^{2}+\left(\Omega_{13}\right)^{2}-\left(\Omega_{8}\right)^{2}-\left(\Omega_{15}\right)^{2} \\
\left(\Omega_{14}\right)^{2}-\left(\Omega_{7}\right)^{2}+\left(\Omega_{16}\right)^{2}-\left(\Omega_{2}\right)^{2}=\left(\Omega_{6}\right)^{2}-\left(\Omega_{13}\right)^{2}+\left(\Omega_{8}\right)^{2}-\left(\Omega_{15}\right)^{2}
\end{gathered}
$$




\section{REFERENCES}

* Research supported in part by the U. S. National Science Foundation.

[1] I. S. Barker, A. Donnachie, and J. K. Storrow, Nucl. Phys. B95, 347 (1975).

[2] G. Keaton and R. Workman, Phys. Rev. C53, 1434 (1996).

[3] M. Pichowsky, Ç. Şavkli and F. Tabakin, Nucl. Phys. A370, 311 (1994).

[4] C. G. Fasano, F. Tabakin, and B. Saghai, Phys. Rev. C46, 2430 (1992).

[5] M. J. Moravcsik, Phys. Rev. D29, 2625 (1984); J. Math. Phys. 26, 211 (1995).

[6] N. W. Dean and P. Lee, Phys. Rev. D5, 2741 (1972).

[7] C. Itzykson and J.-B. Zuber, Quantum Field Theory, (McGraw-Hill, New York, 1985), p. 161; M. E. Peskin and D. V. Schroeder, An Introduction to Quantum Field Theory, (Addison-Wesley, Reading, 1995), p. 75.

[8] G. R. Goldstein, J. F. Owens III, J. P Rutherfoord, and M. J. Moravcsik, Nucl. Phys. B80, 164 (1974). 


\section{TABLES}

TABLE I. Spin Observables: The 16 spin observables are expressed in helicity representation and BHP forms. Here they are classified into four type sets: type $\mathcal{S}$ for the differential cross section and single spin observables, and types $\mathcal{B} \mathcal{T}, \mathcal{B R}$ and $\mathcal{T} \mathcal{R}$ for beam-target, beam-recoil and target-recoil spin observables, respectively.

\begin{tabular}{|c|c|c|c|c|}
\hline Spin & Helicity & Transversity & BHP & Set \\
\hline Observable & Representation & Representation & Form & \\
\hline$\check{\Omega}^{1} \equiv \mathcal{I}(\theta)$ & $\frac{1}{2}\left(\left|H_{1}\right|^{2}+\left|H_{2}\right|^{2}+\left|H_{3}\right|^{2}+\left|H_{4}\right|^{2}\right)$ & $\frac{1}{2}\left(\left|b_{1}\right|^{2}+\left|b_{2}\right|^{2}+\left|b_{3}\right|^{2}+\left|b_{4}\right|^{2}\right)$ & $\frac{1}{2}\left\langle b\left|\widetilde{\Gamma}^{1}\right| b\right\rangle$ & \\
\hline$\check{\Omega}^{4} \equiv \check{\Sigma}$ & $\operatorname{Re}\left(-H_{1} H_{4}^{*}+H_{2} H_{3}^{*}\right)$ & $\frac{1}{2}\left(\left|b_{1}\right|^{2}+\left|b_{2}\right|^{2}-\left|b_{3}\right|^{2}-\left|b_{4}\right|^{2}\right)$ & $\frac{1}{2}\left\langle b\left|\widetilde{\Gamma}^{4}\right| b\right\rangle$ & $\mathcal{S}$ \\
\hline$\check{\Omega}^{10} \equiv-\check{T}$ & $\operatorname{Im}\left(H_{1} H_{2}^{*}+H_{3} H_{4}^{*}\right)$ & $\frac{1}{2}\left(-\left|b_{1}\right|^{2}+\left|b_{2}\right|^{2}+\left|b_{3}\right|^{2}-\left|b_{4}\right|^{2}\right)$ & $\frac{1}{2}\left\langle b\left|\widetilde{\Gamma}^{10}\right| b\right\rangle$ & \\
\hline$\check{\Omega}^{12} \equiv \check{P}$ & $\operatorname{Im}\left(-H_{1} H_{3}^{*}-H_{2} H_{4}^{*}\right)$ & $\frac{1}{2}\left(-\left|b_{1}\right|^{2}+\left|b_{2}\right|^{2}-\left|b_{3}\right|^{2}+\left|b_{4}\right|^{2}\right)$ & $\frac{1}{2}\left\langle b\left|\widetilde{\Gamma}^{12}\right| b\right\rangle$ & \\
\hline$\check{\Omega}^{3} \equiv \check{G}$ & $\operatorname{Im}\left(H_{1} H_{4}^{*}-H_{3} H_{2}^{*}\right)$ & $\operatorname{Im}\left(-b_{1} b_{3}^{*}-b_{2} b_{4}^{*}\right)$ & $\frac{1}{2}\left\langle b\left|\widetilde{\Gamma}^{3}\right| b\right\rangle$ & \\
\hline$\check{\Omega}^{5} \equiv \check{H}$ & $\operatorname{Im}\left(-H_{2} H_{4}^{*}+H_{1} H_{3}^{*}\right)$ & $\operatorname{Re}\left(b_{1} b_{3}^{*}-b_{2} b_{4}^{*}\right)$ & $\frac{1}{2}\left\langle b\left|\widetilde{\Gamma}^{5}\right| b\right\rangle$ & $\mathcal{B T}$ \\
\hline$\check{\Omega}^{9} \equiv \check{E}$ & $\frac{1}{2}\left(\left|H_{1}\right|^{2}-\left|H_{2}\right|^{2}+\left|H_{3}\right|^{2}-\left|H_{4}\right|^{2}\right)$ & $\operatorname{Re}\left(b_{1} b_{3}^{*}+b_{2} b_{4}^{*}\right)$ & $\frac{1}{2}\left\langle b\left|\widetilde{\Gamma}^{9}\right| b\right\rangle$ & \\
\hline$\check{\Omega}^{11} \equiv \check{F}$ & $\operatorname{Re}\left(-H_{2} H_{1}^{*}-H_{4} H_{3}^{*}\right)$ & $\operatorname{Im}\left(b_{1} b_{3}^{*}-b_{2} b_{4}^{*}\right)$ & $\frac{1}{2}\left\langle b\left|\widetilde{\Gamma}^{11}\right| b\right\rangle$ & \\
\hline$\check{\Omega}^{14} \equiv \check{O_{x}}$ & $\operatorname{Im}\left(-H_{2} H_{1}^{*}+H_{4} H_{3}^{*}\right)$ & $\operatorname{Re}\left(-b_{1} b_{4}^{*}+b_{2} b_{3}^{*}\right)$ & $\frac{1}{2}\left\langle b\left|\widetilde{\Gamma}^{14}\right| b\right\rangle$ & \\
\hline$\check{\Omega}^{7} \equiv-\check{O_{z}}$ & $\operatorname{Im}\left(H_{1} H_{4}^{*}-H_{2} H_{3}^{*}\right)$ & $\operatorname{Im}\left(-b_{1} b_{4}^{*}-b_{2} b_{3}^{*}\right)$ & $\frac{1}{2}\left\langle b\left|\widetilde{\Gamma}^{7}\right| b\right\rangle$ & $\mathcal{B R}$ \\
\hline$\check{\Omega}^{16} \equiv-\check{C_{x}}$ & $\operatorname{Re}\left(H_{2} H_{4}^{*}+H_{1} H_{3}^{*}\right)$ & $\operatorname{Im}\left(b_{1} b_{4}^{*}-b_{2} b_{3}^{*}\right)$ & $\frac{1}{2}\left\langle b\left|\widetilde{\Gamma}^{16}\right| b\right\rangle$ & \\
\hline$\check{\Omega}^{2} \equiv-\check{C}_{z}$ & $\frac{1}{2}\left(\left|H_{1}\right|^{2}+\left|H_{2}\right|^{2}-\left|H_{3}\right|^{2}-\left|H_{4}\right|^{2}\right)$ & $\operatorname{Re}\left(b_{1} b_{4}^{*}+b_{2} b_{3}^{*}\right)$ & $\frac{1}{2}\left\langle b\left|\widetilde{\Gamma}^{2}\right| b\right\rangle$ & \\
\hline$\check{\Omega}^{6} \equiv-\check{T}_{x}$ & $\operatorname{Re}\left(-H_{1} H_{4}^{*}-H_{2} H_{3}^{*}\right)$ & $\operatorname{Re}\left(-b_{1} b_{2}^{*}+b_{3} b_{4}^{*}\right)$ & $\frac{1}{2}\left\langle b\left|\widetilde{\Gamma}^{6}\right| b\right\rangle$ & \\
\hline$\check{\Omega}^{13} \equiv-\check{T}_{z}$ & $\operatorname{Re}\left(-H_{1} H_{2}^{*}+H_{4} H_{3}^{*}\right)$ & $\operatorname{Im}\left(b_{1} b_{2}^{*}-b_{3} b_{4}^{*}\right)$ & $\frac{1}{2}\left\langle b\left|\widetilde{\Gamma}^{13}\right| b\right\rangle$ & $\mathcal{T R}$ \\
\hline$\check{\Omega}^{8} \equiv \check{L_{x}}$ & $\operatorname{Re}\left(H_{2} H_{4}^{*}-H_{1} H_{3}^{*}\right)$ & $\operatorname{Im}\left(-b_{1} b_{2}^{*}-b_{3} b_{4}^{*}\right)$ & $\frac{1}{2}\left\langle b\left|\widetilde{\Gamma}^{8}\right| b\right\rangle$ & \\
\hline$\check{\Omega}^{15} \equiv \check{L_{z}}$ & $\frac{1}{2}\left(-\left|H_{1}\right|^{2}+\left|H_{2}\right|^{2}+\left|H_{3}\right|^{2}-\left|H_{4}\right|^{2}\right)$ & $\operatorname{Re}\left(-b_{1} b_{2}^{*}-b_{3} b_{4}^{*}\right)$ & $\frac{1}{2}\left\langle b\left|\widetilde{\Gamma}^{15}\right| b\right\rangle$ & \\
\hline
\end{tabular}


TABLE II. Result of linear $(L)$ and antilinear $(A)$ ambiguity transformations applied to observables. The observables are either invariant $(+)$ or change sign $(-)$ under these transformations.

\begin{tabular}{|c|c|c|c|c|c|c|c|c|}
\hline \multirow[b]{2}{*}{ Observable } & \multicolumn{3}{|c|}{$\begin{array}{l}\text { Linear Transformation } L \\
\qquad b_{i} \longrightarrow b_{i}^{\prime}=L_{i j} b_{j}\end{array}$} & \multicolumn{4}{|c|}{$\begin{array}{l}\text { Antilinear Transformation } A \\
\qquad b_{i} \longrightarrow b_{i}^{\prime}=A_{i j} b_{j}^{*}\end{array}$} & \multirow[t]{2}{*}{ Set } \\
\hline & $\widetilde{\Gamma}_{4}$ & $\widetilde{\Gamma}_{10}$ & $\widetilde{\Gamma}_{12}$ & $\widetilde{\Gamma}_{6}$ & $\widetilde{\Gamma}_{8}$ & $\widetilde{\Gamma}_{13}$ & $\widetilde{\Gamma}_{15}$ & \\
\hline$\sigma(\theta)$ & + & + & + & + & + & + & + & \\
\hline$\Sigma$ & + & + & + & + & + & + & + & $\mathcal{S}$ \\
\hline$T$ & + & + & + & + & + & + & + & \\
\hline$P$ & + & + & + & + & + & + & + & \\
\hline$G$ & - & - & + & + & - & + & - & \\
\hline$H$ & - & - & + & - & + & - & + & $\mathcal{B} \mathcal{T}$ \\
\hline$E$ & - & - & + & - & + & - & + & \\
\hline$F$ & - & - & + & + & - & + & - & \\
\hline$O_{x}$ & - & + & - & - & - & + & + & \\
\hline$O_{z}$ & - & + & - & + & + & - & - & $\mathcal{B R}$ \\
\hline$C_{x}$ & - & + & - & + & + & - & - & \\
\hline$C_{z}$ & - & + & - & - & - & + & + & \\
\hline$T_{x}$ & + & - & - & + & - & - & + & \\
\hline$T_{z}$ & + & - & - & - & + & + & - & $\mathcal{T} \mathcal{R}$ \\
\hline$L_{x}$ & + & - & - & - & + & + & - & \\
\hline$L_{z}$ & + & - & - & + & - & - & + & \\
\hline
\end{tabular}


TABLE III. Tables III-VIII enumerate all situations under which four double spin observables, along with the set $\mathcal{S}$, can completely determine the transversity amplitudes. In these tables, ' $\mathbf{X}$ 's' indicate three initially selected measurements, and 'O's' indicate the possible choices for fourth observable that can resolve all the ambiguities.

\begin{tabular}{|c|c|c|c|c|c|c|c|c|c|c|c|c|c|c|c|c|c|c|c|c|c|c|c|c|c|}
\hline$G$ & $\mathrm{X}$ & $\mathbf{X}$ & $\mathbf{X}$ & $\mathbf{X}$ & $\mathbf{X}$ & $\mathbf{X}$ & $\mathbf{X}$ & $\mathbf{X}$ & $\mathrm{X}$ & $\mathbf{X}$ & $\mathbf{X}$ & $\mathrm{X}$ & $\mathbf{X}$ & $\mathbf{x}$ & $\mathbf{X}$ & $\mathbf{X}$ & $\mathrm{X}$ & $\mathbf{x}$ & $\mathbf{X}$ & $\mathbf{x}$ & $\mathbf{x}$ & $\mathbf{x}$ & $\mathbf{X}$ & $\mathbf{X}$ & \\
\hline$H$ & $\mathbf{X}$ & $\mathbf{X}$ & $\mathbf{x}$ & $\mathbf{X}$ & $\mathbf{X}$ & $\mathrm{X}$ & $\mathrm{X}$ & $\mathbf{X}$ & & & & & & & & & & & & & & & & & $\mathcal{B} \mathcal{T}$ \\
\hline$E$ & & & & & & & & & $\mathbf{X}$ & $\mathbf{X}$ & $\mathrm{X}$ & $\mathbf{X}$ & $\mathbf{X}$ & $\mathbf{X}$ & $\mathrm{X}$ & $\mathbf{x}$ & & & & & & & & & \\
\hline$F$ & & & & & & & & & & & & & & & & & $\mathbf{X}$ & $\mathbf{X}$ & $\mathbf{X}$ & $\mathbf{X}$ & $\mathbf{X}$ & $\mathrm{X}$ & $\mathbf{X}$ & $\mathbf{X}$ & \\
\hline$O_{x}$ & $\mathbf{X}$ & & $\mathrm{O}$ & & $\mathrm{O}$ & $\mathrm{O}$ & $\mathrm{O}$ & $\mathrm{O}$ & $\mathbf{X}$ & $\mathrm{O}$ & $\mathrm{O}$ & $\mathrm{O}$ & $\mathrm{O}$ & $\mathrm{O}$ & O & $\mathrm{O}$ & $\mathbf{X}$ & & $\mathrm{O}$ & & $\mathrm{O}$ & & & $\mathrm{O}$ & \\
\hline$O_{z}$ & & $\mathbf{X}$ & & $\mathrm{O}$ & O & O & O & $\mathrm{O}$ & $\mathrm{O}$ & $\mathbf{X}$ & O & $\mathrm{O}$ & $\mathrm{O}$ & O & $\mathrm{O}$ & O & & $\mathbf{X}$ & & $\mathrm{O}$ & & O & O & & $\mathcal{B R}$ \\
\hline$C_{x}$ & $\mathrm{O}$ & & $\mathbf{X}$ & & $\mathrm{O}$ & $\mathrm{O}$ & $\mathrm{O}$ & $\mathrm{O}$ & $\mathrm{O}$ & $\mathrm{O}$ & $\mathbf{X}$ & $\mathrm{O}$ & $\mathrm{O}$ & $\mathrm{O}$ & $\mathrm{O}$ & $\mathrm{O}$ & $\mathrm{O}$ & & $\mathbf{X}$ & & & $\mathrm{O}$ & $\mathrm{O}$ & & \\
\hline$C_{z}$ & & $\mathrm{O}$ & & $\mathrm{X}$ & $\mathrm{O}$ & O & $\mathrm{O}$ & $\mathrm{O}$ & $\mathrm{O}$ & $\mathrm{O}$ & $\mathrm{O}$ & $\mathbf{X}$ & $\mathrm{O}$ & O & $\mathrm{O}$ & $\mathrm{O}$ & & $\mathbf{O}$ & & $\mathbf{X}$ & $\mathrm{O}$ & & & $\mathrm{O}$ & \\
\hline$T_{x}$ & $\mathrm{O}$ & $\mathrm{O}$ & $\mathrm{O}$ & $\mathrm{O}$ & $\mathbf{X}$ & $\mathrm{O}$ & $\mathrm{O}$ & $\mathrm{O}$ & $\mathrm{O}$ & $\mathrm{O}$ & $\mathrm{O}$ & $\mathrm{O}$ & $\mathbf{X}$ & & $\mathrm{O}$ & & $\mathrm{O}$ & & & $\mathrm{O}$ & $\mathrm{X}$ & & $\mathrm{O}$ & & \\
\hline$T_{z}$ & $\mathrm{O}$ & $\mathrm{O}$ & O & $\mathrm{O}$ & $\mathrm{O}$ & $\mathrm{X}$ & $\mathrm{O}$ & $\mathrm{O}$ & $\mathrm{O}$ & $\mathrm{O}$ & $\mathrm{O}$ & $\mathrm{O}$ & & $\mathbf{X}$ & & O & & $\mathrm{O}$ & $\mathrm{O}$ & & & $\mathrm{X}$ & & $\mathrm{O}$ & $\mathcal{T R}$ \\
\hline$L_{x}$ & $\mathrm{O}$ & O & $\mathrm{O}$ & O & O & O & $\mathbf{X}$ & $\mathrm{O}$ & $\mathrm{O}$ & $\mathrm{O}$ & $\mathrm{O}$ & $\mathbf{O}$ & $\mathrm{O}$ & & $\mathbf{X}$ & & & $\mathbf{O}$ & O & & O & & $\mathbf{X}$ & & \\
\hline$L_{z}$ & $\mathrm{O}$ & $\mathrm{O}$ & $\mathrm{O}$ & $\mathrm{O}$ & $\mathrm{O}$ & $\mathrm{O}$ & $\mathrm{O}$ & $\mathbf{X}$ & $\mathrm{O}$ & $\mathrm{O}$ & $\mathrm{O}$ & $\mathrm{O}$ & & $\mathrm{O}$ & & $\mathbf{X}$ & $\mathrm{O}$ & & & $\mathrm{O}$ & & $\mathrm{O}$ & & $\mathbf{X}$ & \\
\hline
\end{tabular}


TABLE IV.

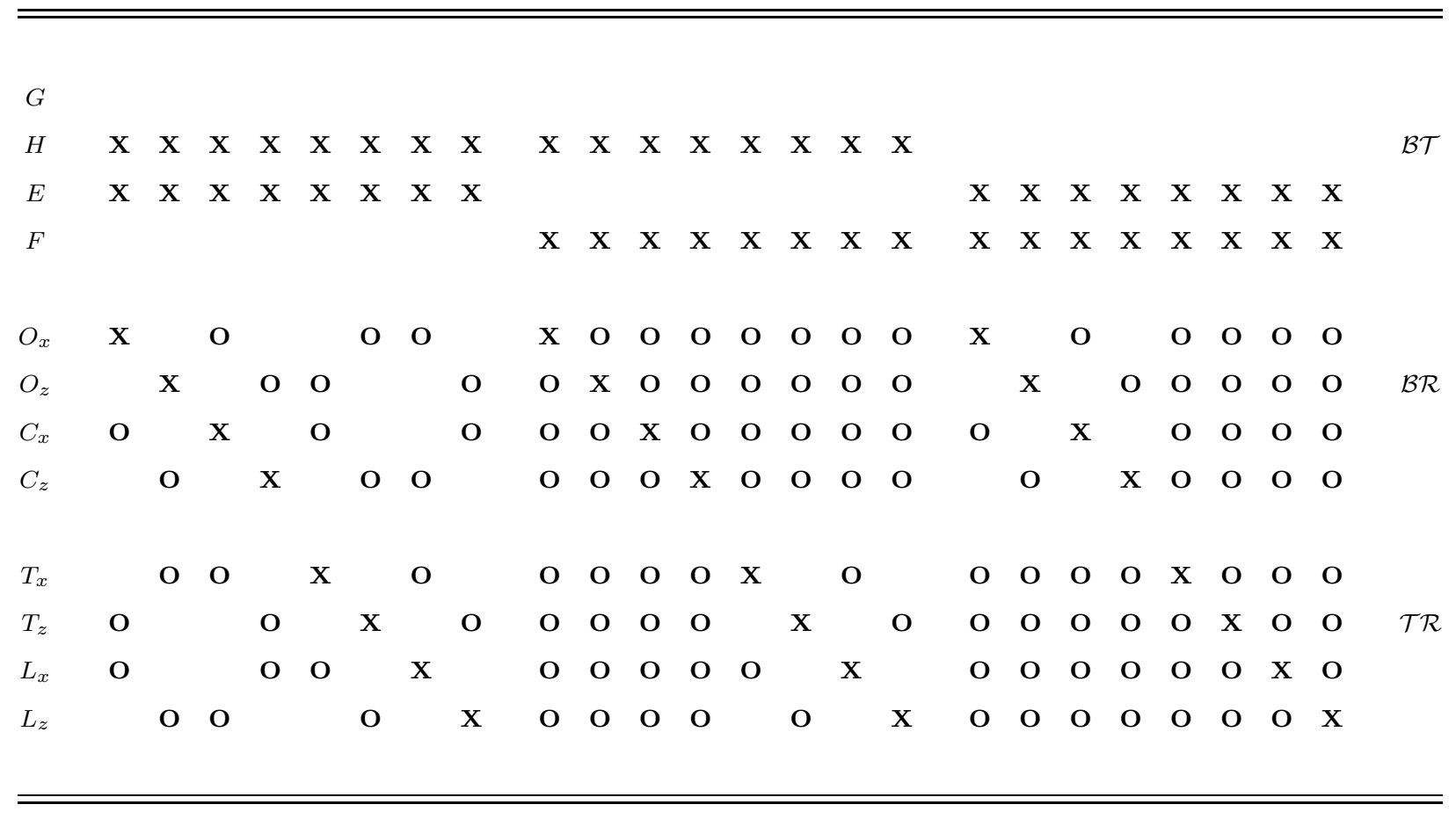

TABLE V.

\begin{tabular}{|c|c|c|c|c|c|c|c|c|c|c|c|c|c|c|c|c|c|c|c|c|c|c|c|c|c|}
\hline$G$ & $\mathrm{X}$ & & O & & $\mathrm{O}$ & $\mathrm{O}$ & $\mathrm{O}$ & $\mathbf{O}$ & $\mathrm{X}$ & $\mathrm{O}$ & $\mathrm{O}$ & $\mathbf{O}$ & $\mathrm{O}$ & $\mathbf{O}$ & $\mathrm{O}$ & $\mathrm{O}$ & $\mathrm{X}$ & & $\mathrm{O}$ & & & $\mathrm{O}$ & $\mathrm{O}$ & & \\
\hline$H$ & & $\mathbf{X}$ & & $\mathrm{O}$ & $\mathrm{O}$ & $\mathrm{O}$ & $\mathrm{O}$ & $\mathrm{O}$ & $\mathrm{O}$ & $\mathrm{x}$ & $\mathrm{O}$ & $\mathrm{O}$ & $\mathrm{O}$ & $\mathrm{O}$ & O & $\mathbf{O}$ & & $\mathbf{X}$ & & $\mathrm{O}$ & $\mathrm{O}$ & & & $\mathrm{O}$ & $\mathcal{B} \mathcal{T}$ \\
\hline$E$ & $\mathrm{O}$ & & $\mathbf{x}$ & & $\mathrm{O}$ & $\mathrm{O}$ & $\mathrm{O}$ & $\mathrm{O}$ & $\mathrm{O}$ & $\mathrm{O}$ & $\mathbf{X}$ & $\mathrm{O}$ & $\mathrm{O}$ & $\mathrm{O}$ & $\mathrm{O}$ & $\mathrm{O}$ & $\mathrm{O}$ & & $\mathbf{X}$ & & $\mathrm{O}$ & & & $\mathrm{O}$ & \\
\hline$F$ & & $\mathrm{O}$ & & $\mathrm{X}$ & $\mathrm{O}$ & $\mathrm{O}$ & $\mathrm{O}$ & $\mathrm{O}$ & $\mathrm{O}$ & $\mathrm{O}$ & $\mathrm{O}$ & $\mathbf{X}$ & $\mathrm{O}$ & O & $\mathrm{O}$ & O & & $\mathrm{O}$ & & $\mathbf{X}$ & & O & O & & \\
\hline$O_{x}$ & $\mathbf{X}$ & $\mathbf{X}$ & $\mathbf{X}$ & $\mathbf{X}$ & $\mathbf{X}$ & $\mathbf{X}$ & $\mathbf{X}$ & $\mathbf{X}$ & $\mathrm{X}$ & $\mathrm{X}$ & $\mathrm{X}$ & $\mathbf{X}$ & $\mathbf{X}$ & $\mathbf{X}$ & $\mathbf{X}$ & $\mathbf{X}$ & $\mathbf{X}$ & $\mathbf{X}$ & $\mathbf{X}$ & $\mathbf{X}$ & $\mathbf{X}$ & $\mathbf{X}$ & $\mathbf{X}$ & $\mathbf{X}$ & \\
\hline$O_{z}$ & $\mathrm{X}$ & $\mathrm{X}$ & $\mathbf{X}$ & $\mathbf{X}$ & $\mathbf{X}$ & $\mathrm{X}$ & $\mathbf{X}$ & $\mathbf{X}$ & & & & & & & & & & & & & & & & & $\mathcal{B R}$ \\
\hline$C_{x}$ & & & & & & & & & $\mathbf{X}$ & $\mathrm{X}$ & $\mathbf{X}$ & $\mathbf{X}$ & $\mathbf{x}$ & $\mathbf{X}$ & $\mathbf{X}$ & $\mathbf{x}$ & & & & & & & & & \\
\hline$C_{z}$ & & & & & & & & & & & & & & & & & $\mathbf{X}$ & $\mathbf{X}$ & $\mathrm{X}$ & $\mathrm{X}$ & $\mathbf{X}$ & X & $\mathbf{X}$ & $\mathrm{x}$ & \\
\hline$T_{x}$ & O & O & O & O & $\mathbf{X}$ & $\mathrm{O}$ & $\mathbf{O}$ & $\mathrm{O}$ & $\mathrm{O}$ & O & $\mathrm{O}$ & $\mathrm{O}$ & $\mathrm{X}$ & O & & & & $\mathbf{O}$ & O & & $\mathbf{X}$ & O & & & \\
\hline$T_{z}$ & $\mathrm{O}$ & $\mathrm{O}$ & O & $\mathrm{O}$ & $\mathrm{O}$ & $\mathrm{X}$ & $\mathrm{O}$ & $\mathrm{O}$ & $\mathrm{O}$ & $\mathrm{O}$ & O & $\mathbf{O}$ & $\mathrm{O}$ & $\mathrm{X}$ & & & $\mathrm{O}$ & & & $\mathrm{O}$ & $\mathrm{O}$ & $\mathbf{X}$ & & & $\mathcal{T R}$ \\
\hline$L_{x}$ & $\mathrm{O}$ & O & $\mathrm{O}$ & O & $\mathrm{O}$ & $\mathrm{O}$ & $\mathrm{X}$ & $\mathrm{O}$ & $\mathrm{O}$ & O & $\mathrm{O}$ & $\mathrm{O}$ & & & $\mathbf{X}$ & O & $\mathrm{O}$ & & & O & & & $\mathbf{X}$ & $\mathrm{O}$ & \\
\hline$L_{z}$ & $\mathrm{O}$ & $\mathrm{O}$ & $\mathrm{O}$ & $\mathrm{O}$ & O & $\mathrm{O}$ & $\mathrm{O}$ & $\mathbf{X}$ & $\mathrm{O}$ & $\mathrm{O}$ & $\mathrm{O}$ & $\mathbf{O}$ & & & O & $\mathrm{X}$ & & $\mathrm{O}$ & $\mathrm{O}$ & & & & O & $\mathbf{X}$ & \\
\hline
\end{tabular}


TABLE VI.

\begin{tabular}{|c|c|c|c|c|c|c|c|c|c|c|c|c|c|c|c|c|c|c|c|c|c|c|c|c|c|}
\hline$G$ & $\mathbf{X}$ & & O & & $\mathrm{O}$ & & & $\mathrm{O}$ & $\mathbf{X}$ & $\mathrm{O}$ & $\mathrm{O}$ & $\mathrm{O}$ & $\mathrm{O}$ & $\mathrm{O}$ & $\mathrm{O}$ & $\mathrm{O}$ & $\mathbf{X}$ & & $\mathrm{O}$ & & $\mathrm{O}$ & $\mathrm{O}$ & $\mathrm{O}$ & $\mathrm{O}$ & \\
\hline$H$ & & $\mathrm{X}$ & & $\mathrm{O}$ & & $\mathrm{O}$ & $\mathrm{O}$ & & $\mathrm{O}$ & $\mathbf{X}$ & $\mathrm{O}$ & $\mathrm{O}$ & $\mathrm{O}$ & $\mathrm{O}$ & $\mathrm{O}$ & $\mathbf{O}$ & & $\mathbf{X}$ & & O & $\mathrm{O}$ & $\mathrm{O}$ & $\mathrm{O}$ & $\mathrm{O}$ & $\mathcal{B} \mathcal{T}$ \\
\hline$E$ & $\mathrm{O}$ & & $\mathbf{X}$ & & & $\mathrm{O}$ & $\mathrm{O}$ & & $\mathrm{O}$ & $\mathrm{O}$ & $\mathbf{X}$ & O & $\mathrm{O}$ & O & $\mathrm{O}$ & $\mathrm{O}$ & $\mathrm{O}$ & & $\mathbf{X}$ & & O & O & O & O & \\
\hline$F$ & & O & & $\mathbf{X}$ & $\mathbf{O}$ & & & $\mathrm{O}$ & $\mathrm{O}$ & $\mathrm{O}$ & O & $\mathrm{X}$ & $\mathrm{O}$ & O & $\mathrm{O}$ & $\mathrm{O}$ & & O & & $\mathbf{X}$ & $\mathrm{O}$ & $\mathrm{O}$ & O & $\mathrm{O}$ & \\
\hline$O_{x}$ & & & & & & & & & & & & & & & & & & & & & & & & & \\
\hline$O_{z}$ & $\mathbf{X}$ & $\mathbf{X}$ & $\mathbf{X}$ & $\mathbf{X}$ & $\mathbf{X}$ & $\mathbf{X}$ & $\mathrm{X}$ & $\mathbf{X}$ & $\mathbf{X}$ & $\mathrm{X}$ & $\mathbf{X}$ & $\mathbf{X}$ & $\mathbf{X}$ & $\mathrm{X}$ & $\mathbf{X}$ & $\mathbf{X}$ & & & & & & & & & $\mathcal{B R}$ \\
\hline$C_{x}$ & $\mathbf{X}$ & $\mathrm{X}$ & $\mathbf{X}$ & $\mathrm{X}$ & $\mathbf{X}$ & $\mathbf{X}$ & $\mathbf{X}$ & $\mathbf{X}$ & & & & & & & & & $\mathbf{X}$ & $\mathbf{X}$ & $\mathbf{X}$ & $\mathbf{X}$ & $\mathbf{X}$ & $\mathbf{x}$ & $\mathbf{X}$ & $\mathbf{X}$ & \\
\hline$C_{z}$ & & & & & & & & & $\mathrm{X}$ & $\mathbf{X}$ & $\mathbf{X}$ & $\mathbf{X}$ & $\mathbf{X}$ & $\mathrm{X}$ & $\mathbf{X}$ & $\mathbf{X}$ & $\mathbf{X}$ & $\mathrm{X}$ & $\mathbf{X}$ & $\mathbf{X}$ & $\mathbf{X}$ & $\mathbf{X}$ & $\mathbf{X}$ & $\mathbf{x}$ & \\
\hline$T_{x}$ & O & & & $\mathrm{O}$ & $\mathbf{X}$ & O & & & $\mathrm{O}$ & O & O & $\mathbf{O}$ & $\mathrm{X}$ & $\mathrm{O}$ & & & O & O & O & O & $\mathbf{X}$ & O & O & O & \\
\hline$T_{z}$ & & O & $\mathbf{O}$ & & $\mathrm{O}$ & $\mathbf{X}$ & & & $\mathrm{O}$ & O & $\mathbf{O}$ & O & $\mathrm{O}$ & $\mathbf{X}$ & & & O & O & O & O & O & $\mathbf{X}$ & $\mathrm{O}$ & O & $\mathcal{T} \mathcal{R}$ \\
\hline$L_{x}$ & & O & $\mathrm{O}$ & & & & $\mathrm{X}$ & $\mathrm{O}$ & $\mathrm{O}$ & $\mathrm{O}$ & O & $\mathrm{O}$ & & & $\mathrm{X}$ & $\mathrm{O}$ & $\mathbf{O}$ & O & O & O & $\mathrm{O}$ & $\mathbf{O}$ & $\mathbf{X}$ & O & \\
\hline$L_{z}$ & O & & & O & & & $\mathrm{O}$ & $\mathbf{X}$ & $\mathrm{O}$ & $\mathbf{O}$ & O & $\mathrm{O}$ & & & O & $\mathbf{X}$ & $\mathrm{O}$ & $\mathbf{O}$ & O & O & $\mathrm{O}$ & $\mathrm{O}$ & $\mathbf{O}$ & $\mathbf{X}$ & \\
\hline
\end{tabular}

\section{TABLE VII.}

\begin{tabular}{|c|c|c|c|c|c|c|c|c|c|c|c|c|c|c|c|c|c|c|c|c|c|c|c|c|c|}
\hline$G$ & $\mathbf{X}$ & $\mathrm{O}$ & & & O & $\mathrm{O}$ & $\mathrm{O}$ & $\mathbf{O}$ & $\mathbf{X}$ & $\mathrm{O}$ & $\mathrm{O}$ & $\mathbf{O}$ & $\mathbf{O}$ & $\mathbf{O}$ & $\mathrm{O}$ & $\mathrm{O}$ & $\mathbf{X}$ & $\mathbf{O}$ & & & & $\mathrm{O}$ & $\mathrm{O}$ & & \\
\hline$H$ & $\mathrm{O}$ & $\mathbf{X}$ & & & $\mathrm{O}$ & $\mathrm{O}$ & $\mathrm{O}$ & $\mathrm{O}$ & $\mathrm{O}$ & $\mathrm{X}$ & $\mathrm{O}$ & $\mathrm{O}$ & $\mathrm{O}$ & $\mathrm{O}$ & O & $\mathrm{O}$ & $\mathrm{O}$ & $\mathbf{X}$ & & & $\mathrm{O}$ & & & $\mathrm{O}$ & $\mathcal{B \mathcal { T }}$ \\
\hline$E$ & & & $\mathbf{X}$ & $\mathrm{O}$ & O & $\mathrm{O}$ & $\mathrm{O}$ & $\mathrm{O}$ & $\mathrm{O}$ & $\mathrm{O}$ & $\mathbf{X}$ & $\mathrm{O}$ & $\mathrm{O}$ & $\mathrm{O}$ & $\mathrm{O}$ & $\mathrm{O}$ & & & $\mathbf{X}$ & $\mathrm{O}$ & $\mathrm{O}$ & & & $\mathrm{O}$ & \\
\hline$F$ & & & O & $\mathbf{X}$ & $\mathrm{O}$ & $\mathrm{O}$ & $\mathrm{O}$ & $\mathrm{O}$ & $\mathrm{O}$ & $\mathrm{O}$ & $\mathrm{O}$ & $\mathrm{X}$ & $\mathrm{O}$ & $\mathrm{O}$ & O & $\mathrm{O}$ & & & $\mathrm{O}$ & $\mathbf{X}$ & & $\mathrm{O}$ & O & & \\
\hline$O_{x}$ & $\mathbf{O}$ & $\mathrm{O}$ & O & $\mathrm{O}$ & $\mathbf{X}$ & $\mathrm{O}$ & $\mathrm{O}$ & $\mathbf{O}$ & $\mathrm{O}$ & $\mathrm{O}$ & $\mathrm{O}$ & $\mathbf{O}$ & $\mathbf{X}$ & $\mathbf{O}$ & & & & $\mathbf{O}$ & $\mathrm{O}$ & & $\mathbf{X}$ & $\mathrm{O}$ & & & \\
\hline$O_{z}$ & O & $\mathrm{O}$ & $\mathrm{O}$ & $\mathrm{O}$ & $\mathrm{O}$ & $\mathbf{X}$ & $\mathrm{O}$ & $\mathrm{O}$ & $\mathrm{O}$ & $\mathrm{O}$ & $\mathrm{O}$ & $\mathrm{O}$ & $\mathrm{O}$ & $\mathbf{X}$ & & & $\mathrm{O}$ & & & O & $\mathrm{O}$ & $\mathbf{X}$ & & & $\mathcal{B R}$ \\
\hline$C_{x}$ & O & $\mathrm{O}$ & O & $\mathrm{O}$ & $\mathrm{O}$ & $\mathrm{O}$ & $\mathbf{X}$ & $\mathrm{O}$ & $\mathrm{O}$ & $\mathrm{O}$ & $\mathrm{O}$ & $\mathrm{O}$ & & & $\mathbf{X}$ & $\mathrm{O}$ & $\mathrm{O}$ & & & O & & & $\mathbf{X}$ & $\mathrm{O}$ & \\
\hline$C_{z}$ & $\mathrm{O}$ & $\mathrm{O}$ & $\mathbf{O}$ & O & $\mathrm{O}$ & $\mathrm{O}$ & $\mathrm{O}$ & $\mathrm{X}$ & $\mathrm{O}$ & $\mathrm{O}$ & $\mathrm{O}$ & $\mathrm{O}$ & & & $\mathrm{O}$ & $\mathrm{X}$ & & O & O & & & & $\mathbf{O}$ & $\mathbf{X}$ & \\
\hline$T_{x}$ & $\mathbf{X}$ & $\mathbf{X}$ & $\mathbf{X}$ & $\mathbf{X}$ & $\mathbf{X}$ & $\mathbf{X}$ & $\mathbf{X}$ & $\mathbf{X}$ & $\mathrm{X}$ & $\mathbf{X}$ & $\mathbf{X}$ & $\mathbf{X}$ & $\mathbf{X}$ & $\mathbf{X}$ & $\mathbf{X}$ & $\mathbf{X}$ & $\mathbf{X}$ & $\mathbf{X}$ & $\mathbf{X}$ & $\mathbf{X}$ & $\mathbf{X}$ & $\mathbf{X}$ & $\mathbf{X}$ & $\mathbf{X}$ & \\
\hline$T_{z}$ & $\mathbf{X}$ & $\mathbf{X}$ & $\mathbf{X}$ & $\mathbf{X}$ & $\mathbf{X}$ & $\mathbf{X}$ & $\mathbf{X}$ & $\mathbf{X}$ & & & & & & & & & & & & & & & & & $\mathcal{T R}$ \\
\hline$L_{x}$ & & & & & & & & & $\mathrm{X}$ & $\mathbf{X}$ & $\mathbf{X}$ & $\mathbf{X}$ & $\mathbf{X}$ & $\mathbf{X}$ & $\mathbf{X}$ & $\mathbf{X}$ & & & & & & & & & \\
\hline$L_{z}$ & & & & & & & & & & & & & & & & & $\mathrm{X}$ & $\mathrm{X}$ & $\mathbf{X}$ & $\mathrm{X}$ & $\mathrm{X}$ & $\mathbf{X}$ & $\mathbf{x}$ & $\mathbf{X}$ & \\
\hline
\end{tabular}


TABLE VIII.

\begin{tabular}{|c|c|c|c|c|c|c|c|c|c|c|c|c|c|c|c|c|c|c|c|c|c|c|c|c|c|}
\hline$G$ & $\mathbf{X}$ & $\mathrm{O}$ & & & $\mathrm{O}$ & & & $\mathrm{O}$ & $\mathbf{X}$ & O & $\mathrm{O}$ & $\mathrm{O}$ & $\mathrm{O}$ & $\mathrm{O}$ & $\mathrm{O}$ & $\mathrm{O}$ & X & O & & & $\mathrm{O}$ & $\mathrm{O}$ & $\mathrm{O}$ & $\mathrm{O}$ & \\
\hline$H$ & $\mathrm{O}$ & $\mathrm{X}$ & & & & $\mathrm{O}$ & $\mathrm{O}$ & & $\mathrm{O}$ & $\mathrm{X}$ & $\mathrm{O}$ & $\mathrm{O}$ & O & $\mathrm{O}$ & $\mathrm{O}$ & $\mathrm{O}$ & $\mathrm{O}$ & $\mathbf{X}$ & & & $\mathrm{O}$ & $\mathrm{O}$ & O & $\mathrm{O}$ & $\mathcal{B T}$ \\
\hline$E$ & & & $\mathbf{X}$ & $\mathrm{O}$ & & $\mathrm{O}$ & $\mathrm{O}$ & & $\mathrm{O}$ & O & $\mathbf{X}$ & O & O & $\mathrm{O}$ & $\mathrm{O}$ & O & & & $\mathbf{X}$ & O & $\mathrm{O}$ & $\mathrm{O}$ & O & O & \\
\hline$F$ & & & O & $\mathbf{X}$ & O & & & $\mathrm{O}$ & O & $\mathrm{O}$ & $\mathrm{O}$ & $\mathbf{X}$ & O & O & $\mathrm{O}$ & $\mathbf{O}$ & & & O & $\mathbf{X}$ & $\mathrm{O}$ & $\mathbf{O}$ & O & $\mathrm{O}$ & \\
\hline$O_{x}$ & O & & & O & $\mathbf{X}$ & $\mathrm{O}$ & & & O & $\mathrm{O}$ & $\mathrm{O}$ & O & $\mathbf{X}$ & O & & & O & O & $\mathrm{O}$ & O & $\mathbf{X}$ & $\mathrm{O}$ & $\mathbf{O}$ & $\mathbf{O}$ & \\
\hline$O_{z}$ & & $\mathbf{O}$ & O & & O & $\mathbf{X}$ & & & $\mathrm{O}$ & O & O & $\mathrm{O}$ & $\mathrm{O}$ & $\mathbf{X}$ & & & $\mathrm{O}$ & $\mathrm{O}$ & O & O & $\mathrm{O}$ & $\mathbf{X}$ & O & $\mathrm{O}$ & $\mathcal{B R}$ \\
\hline$C_{x}$ & & $\mathbf{O}$ & O & & & & $\mathbf{X}$ & O & O & $\mathrm{O}$ & O & O & & & $\mathbf{X}$ & $\mathbf{O}$ & O & O & O & O & $\mathrm{O}$ & $\mathbf{O}$ & $\mathbf{X}$ & $\mathbf{O}$ & \\
\hline$C_{z}$ & $\mathrm{O}$ & & & $\mathrm{O}$ & & & $\mathrm{O}$ & $\mathbf{X}$ & O & $\mathbf{O}$ & O & $\mathrm{O}$ & & & $\mathrm{O}$ & $\mathbf{X}$ & O & O & O & O & $\mathbf{O}$ & $\mathrm{O}$ & O & $\mathbf{X}$ & \\
\hline$T_{x}$ & & & & & & & & & & & & & & & & & & & & & & & & & \\
\hline$T_{z}$ & X & $\mathbf{X}$ & $\mathbf{X}$ & $\mathbf{X}$ & $\mathbf{X}$ & $\mathbf{X}$ & $\mathbf{X}$ & $\mathbf{X}$ & $\mathbf{X}$ & $\mathbf{X}$ & $\mathbf{X}$ & $\mathbf{X}$ & $\mathrm{X}$ & $\mathrm{X}$ & $\mathbf{X}$ & $\mathbf{X}$ & & & & & & & & & $\mathcal{T} \mathcal{R}$ \\
\hline$L_{x}$ & $\mathbf{X}$ & $\mathbf{X}$ & $\mathbf{X}$ & $\mathbf{X}$ & $\mathbf{X}$ & $\mathrm{X}$ & $\mathbf{X}$ & $\mathbf{X}$ & & & & & & & & & $\mathbf{X}$ & $\mathbf{X}$ & $\mathbf{X}$ & $\mathrm{X}$ & $\mathbf{X}$ & $\mathbf{X}$ & $\mathbf{X}$ & $\mathbf{X}$ & \\
\hline$L_{z}$ & & & & & & & & & $\mathbf{X}$ & $\mathbf{X}$ & $\mathbf{X}$ & $\mathbf{X}$ & $\mathrm{X}$ & $\mathbf{X}$ & $\mathbf{X}$ & $\mathbf{X}$ & $\mathbf{X}$ & $\mathbf{X}$ & $\mathbf{X}$ & $\mathbf{X}$ & $\mathrm{X}$ & $\mathbf{X}$ & $\mathbf{X}$ & $\mathbf{X}$ & \\
\hline
\end{tabular}

